\title{
Inverse and Approximation Problem for Two-Dimensional Fractal Sets
}

\author{
Roberto Rinaldo, Member, IEEE, and Avideh Zakhor, Member, IEEE
}

\begin{abstract}
The geometry of fractals is rich enough that they have extensively been used to model natural phenomena and images. Iterated function systems (IFS) theory provides a convenient way to describe and classify deterministic fractals in the form of a recursive definition. As a result, it is conceivable to develop image representation schemes based on the IFS parameters that correspond to a given fractal image. In this paper, we consider two distinct problems: an inverse problem and an approximation problem. The inverse problem involves finding the IFS parameters of a signal that is exactly generated via an IFS. We make use of the wavelet transform and of the image moments to solve the inverse problem. The approximation problem involves finding a fractal IFS-generated image whose moments match, either exactly or in a mean squared error sense, a range of moments of the original image. The approximating measures are generated by an IFS model of a special form and provide a general basis for the approximation of arbitrary images. Experimental results verifying our approach will be presented.
\end{abstract}

\section{INTRODUCTION}

F RACTAL geometry has been shown to provide statistical and deterministic models for a large class of images that represent natural objects and scenery. As a result, fractal modeling has been applied to diverse problems such as texture segmentation, image compression, and the rendering of natural scenery in computer graphics. From a visual point of view, fractal images share the common property that they exhibit a great deal of self similarity across different scales. Thus, if we were to look at a fractal object through a magnifying lens, we would see the same structures at different resolutions. From a mathematical point of view, the main characteristic of fractal objects is that a small number of parameters is sufficient for their representation [1].

In this paper, we are concerned with compact representation of fractal images or self similar images via iterated function systems (IFS). In particular, we consider two distinct problems: 1) the inverse problem to find the IFS parameters of a signal that is exactly generated via an IFS and 2) the approximation problem to model arbitrary self similar signals with the IFS parameters. Although the latter problem is of more practical importance, as we will see in this paper, the

Manuscript received January 30, 1993; revised August 10, 1993. This work was supported by the National Science Foundation PYI award MIP-9057466 and Office of Naval Research Young Investigator Award N00014-92-J-1732 The associate editor coordinating the review of this paper and approving it for publication was Prof. Rama Chellappa.

R. Rinaldo was with the Department of Electrical Engineering, University of California, Berkeley, CA, 94720 USA. He is now with the Dipartimento di Elettronica e Informatica, University of Padova, Padova, Italy.

A. Zakhor is with the Department of Electrical Engineering, University of California, Berkeley, CA, 94720 USA

IEEE Log Number 9404448. solution of the inverse problem is instrumental in our approach to the approximation problem.

Our basic approach to the solution of these problems is based on the moment method. For the inverse problem, the image moments depend on the IFS parameters and are used in a procedure that allows us to retrieve them. On the other hand, the goal of the approximation procedure is to find a fractal model that generates an image whose moments match a finite number of moments of the original image or are close to them in a mean squared sense. A set of constraints must be satisfied by the original image for the exact matching procedure to be successful. The approximating measures are generated by an IFS model of a special form and provide a general basis for the approximation of arbitrary images.

Another tool that is used for the solution of the inverse problem is the wavelet transform. One of the major applications of the wavelet transform is multiresolution signal analysis, and it is conceivable that it can be used to determine the parameters of a fractal object. Indeed, it has been shown that the redundancy of a fractal signal with respect to scale variation is mirrored by its wavelet decomposition in that the wavelet transform at a finer scale is obtainable from the wavelet transform at coarser scales [5]. In this paper, we exploit this fact to determine the defining parameters of a fractal signal from the decay of the wavelet coefficients among scales and from the moments of the measure associated with the image.

The outline of the paper is as follows. In Section II, we review IFS theory. Section III describes the use of the wavelet transform and the moment method in solving the inverse problem. Section IV includes experimental results of the technique of Section III for IFS-generated images. Section V presents the approximation procedure for arbitrary images, and in Section VI, we present approximation examples. Section VII is the conclusion.

\section{A REVIEW OF ITERATEd FUnCTION Systems}

In this section, we introduce basic results regarding the use of IFS for the generation of fractal images. As we will see, IFS with probabilities can be used to generate gray-tone images, whose support is a fractal object. Specifically, the image renders the "invariant measure" associated with the IFS.

Given a complete metric space $(\mathbf{X}, d)$, where $d$ denotes a metric in $\mathbf{X}$, we can define the metric space $(\mathcal{H}(\mathbf{X}), h)$, where $\mathcal{H}(\mathbf{X})$ is the space of compact subsets of $\mathbf{X}$, and the distance $h$ between two sets $A$ and $B$ is the Hausdorff distance, which is characterized in terms of the metric $d$. Under these 
conditions, it can be shown that the metric space $\mathcal{H}(\mathbf{X})$ is complete according to the Hausdorff metric [1].

IFS theory defines a fractal to be the fixed point of a contraction mapping on $\mathcal{H}(\mathbf{X})$ with respect to the Hausdorff metric. Specifically, the map is defined as

$$
w(B) \triangleq \bigcup_{i=1}^{N} w_{i}(B)
$$

where $\left\{w_{1}, \ldots, w_{N}\right\}$ are contraction maps $w_{i}: \mathbf{X} \rightarrow \mathbf{X}$, and $B$ is a set. $\left\{\mathbf{X}, w_{1}, \ldots, w_{N}\right\}$ is called a hyperbolic IFS, or IFS for short. The maps $w_{1}, \ldots, w_{N}$ can be chosen to be affine transformations on $\mathrm{X}=R^{2}$ defined by a matrix $Q_{i}$ and a displacement vector $\underline{d}_{i}$ :

$$
w_{i}(\underline{x})=Q_{i} \underline{x}+\underline{d}_{i}=\left[\begin{array}{ll}
q_{i 11} & q_{i 12} \\
q_{i 21} & q_{i 22}
\end{array}\right]\left[\begin{array}{l}
x \\
y
\end{array}\right]+\left[\begin{array}{l}
d_{1_{i}} \\
d_{2_{i}}
\end{array}\right] .
$$

IFS representation is quite powerful in that successive application of the map (1) to any subset $B$ of $\mathrm{X}=R^{2}$ converges to a unique subset of $\mathbf{X}$ called the attractor of the IFS system [1]. More precisely, we have

$$
A=\lim _{n \rightarrow+\infty} w^{\circ n}(B)
$$

where $A$ is the attractor, and $w^{\circ n}$ denotes $n$ iterations of the map $w$. Thus, $w^{\circ 0}(B)=B$ and $w^{\circ(n+1)}(B)=w\left(w^{\circ n}(B)\right)$. Since the attractor is the fixed point of the transformation, it is invariant under the transformation itself, and therefore, it is equal to the union of $N$ copies of itself transformed by $w_{1}, \ldots, w_{N}$. This invariance leads to the notion that a fractal object is equal to a magnified copy of one of its parts or, in other words, to the notion of self similarity and, more precisely, self affinity if the maps are affine transformations.

A more general definition of IFS is that of IFS with probabilities. In this context, the gray-tone fractal image is modeled as a probability measure over its region of support, and the fractal object is defined as the fixed point of a contractive mapping ${ }^{1}$ on the space of the probability measures $\mathcal{P}(\mathbf{X})$ on a compact space $\mathbf{X}$ [1]. Specifically, if a set of probabilities $p_{1}, \ldots, p_{N}, 0<p_{i}<1, \sum_{i} p_{i}=1$ is associated with the transformations $w_{1}, \ldots, w_{N}$, then the contractive mapping is defined by the Markov operator $\mathcal{M}$ given by

$$
\begin{aligned}
\mathcal{M} \mu_{(n-1)}(B) & =\mu_{(n)}(B) \\
& =\sum_{i=1}^{N} p_{i} \mu_{(n-1)}\left(w_{i}^{-1}(B)\right)
\end{aligned}
$$

where $\mu_{(n)}(B)$ is the probability of the set $B$ at iteration $n$, and $\mathcal{M} \mu_{(n-1)}$ is the Markov operator that maps $\mu_{(n-1)}$ to $\mu_{(n)}$. If $\left\{\mathbf{X}, w_{1}, \ldots, w_{N}\right\}$ is a hyperbolic IFS, then it is possible to show that there is a unique, attractive stationary probability distribution with support equal to the attractor of the IFS. This means that successive application of the Markov operator $\mathcal{M}$ to an arbitrary initial distribution $\nu$ converges in distribution to the measure $\mu$ :

$$
\mathcal{M} \mu=\lim _{n \rightarrow+\infty} \mathcal{M}^{\text {on }}(\nu)=\mu .
$$

\footnotetext{
${ }^{1}$ with respect to the Hutchinson metric.
}

Furthermore, it is possible to show [1] that for any continuous function $f$, we have

$$
\int f(x) d \mu=\sum_{i=1}^{N} p_{i} \int f\left(w_{i}(x)\right) d \mu .
$$

Equation (5) is a direct consequence of the invariance of the measure $\mu$ as shown in (4) and the definition of the Markov operator defined in (3).

The above concepts can be interpreted in the following way. The digital image of the fractal renders the invariant measure, and the normalized value of each pixel $B$ corresponds to the probability of the Borel subset $B$ of $R^{2}$. The invariant measure is obtained by applying the Markov operator (3) iteratively, starting from any given measure, i.e. from any arbitrary graytone image. The definition of the Markov operator in (3) says that the measure of a set $B$ corresponding to a pixel in the image $\mu_{(n)}$ is approximately equal to the scaled sum of the measures of the pixels in $\mu_{(n-1)}$ whose centers are mapped onto $B$ by the transformations $w_{i}$; the scaling factor for the measure of a particular pixel in $\mu_{(n-1)}$ is the probability associated with the transformation that maps it onto $B$. Thus, the new measure $\mu_{(n)}$ is obtained by applying transformations $w_{i}, i=1, \ldots, N$, to the coordinates of each pixel in $\mu_{(n-1)}$ and adding up the scaled measures associated with each pixel in $\mu_{(n)}$.

An alternative dynamical argument can be used to define the invariant measure associated with the Markov operator (3). In this case, the fractal object is modeled as the invariant distribution of a Markov process with transition probability [2]

$$
p(x, B)=\sum_{i=1}^{N} p_{i} \delta_{w_{i}(x)}(B)
$$

where

$$
\delta_{x}(B)= \begin{cases}1 & \text { if } x \in B \\ 0 & \text { otherwise }\end{cases}
$$

In the "chaos game," [1] the measure is rendered by plotting the sequence $\left\{x_{n}\right\}$, where $x_{n+1}$ is obtained by applying to $x_{n}$ one of the $N$ transformations $w_{1}, \ldots, w_{N}$ chosen with probability $p_{i}$. An ergodic theorem [2] assures that the resulting trajectory is dense in the attractor, and the frequency of visit of a region is proportional to its probability, according to the invariant measure defined by (4).

\section{Procedure for the Inverse Problem}

In this section, we describe a way of recovering IFS parameters of a class of fractal signals whose IFS transformations are of the form:

$$
w_{i}(\underline{x}) \triangleq \underline{y}=Q_{i} \underline{x}+\underline{d}_{i}=\left[\begin{array}{cc}
\lambda_{x} & 0 \\
0 & \lambda_{y}
\end{array}\right]\left[\begin{array}{l}
x \\
y
\end{array}\right]+\left[\begin{array}{l}
d_{1_{i}} \\
d_{2_{i}}
\end{array}\right]
$$

with probabilities $p_{i}, i=1, \ldots, N$. We refer to this procedure as an inverse problem because the signal is assumed to be exactly generated by an IFS. As we will see, in Section V we consider the approximation of arbitrary images with an IFS model. Thus, the inherent difference between the inverse and the approximation problem is that the former assumes that the 


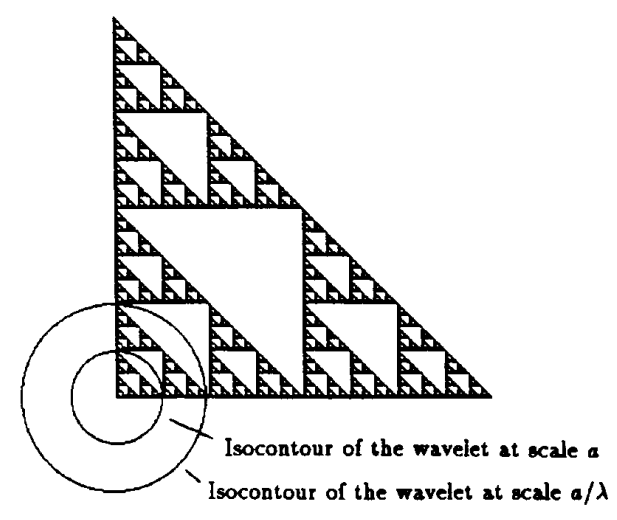

Fig. 1. Sierpinsky triangle and isocontours of the analyzing wavelet.

signal is exactly generated by an IFS, whereas the latter does not.

An example of a fractal signal generated with an IFS of the form (6), which is referred to as the Sierpinsky triangle [1], is shown in Fig. 1. The three transformations $w_{i}$ for the Sierpinsky triangle are defined by $\lambda_{x}=\lambda_{y}=1 / 2$, $p_{i}=p=1 / 3$ and $d_{1_{1}}=0, d_{2_{1}}=0 ; d_{1_{2}}=1 / 2, d_{2_{2}}=0$; $d_{1_{3}}=0, d_{2_{3}}=1 / 2$. As seen, there is a great deal of self similarity in the image of Fig. 1 across various scales.

Our approach to the inverse problem solution is to determine the scaling parameters $\lambda_{x}$ and $\lambda_{y}$ using the wavelet transform as described in Section III-A and then to use the moment method to find the displacement vectors and probabilities, as explained in Section III-B.

\section{A. Determination of the Scaling Parameters Using the Wavelet Transform}

We consider the problem of recovering the parameters $\lambda_{x}$ and $\lambda_{y}$ of the IFS (6) from the wavelet transform of the image $\mu$. We first study the case $\lambda_{x}=\lambda_{y}=\lambda$ in Section III-A-1. Then, we move to the case $\lambda_{x} \neq \lambda_{y}$ in Section III-A-2.

I) Case $\lambda_{x}=\lambda_{y}=\lambda$ : The wavelet transform of the invariant measure $\mu$ associated with the attractor of the IFS in (6) is defined as [6]

$$
W(a, \underline{b})=\int \psi\left(\frac{\underline{x}-\underline{b}}{a}\right) d \mu
$$

where $\underline{x}=(x, y)^{T}, \underline{b}=\left(b_{1}, b_{2}\right)^{T}$ is the point in the attractor where the wavelet is centered, and $a$ denotes the scale. As $a$ becomes smaller, the wavelet "shrinks" and zooms on the details of the fractal measure. Suppose now that $\lambda$ is not known and $p_{i}=p, \forall i$. If one analyzes the fractal object with a wavelet centered at a point $\left(b_{1}, b_{2}\right)$ and changes the scale of the analyzing wavelet from $a$ to $\lambda a$, one obtains a value of the wavelet transform scaled by $p$ [6]. This is because when the analyzing wavelet is shrunken by $\lambda$, the portion of the fractal under the wavelet is similar to the portion under the wavelet before the wavelet was shrunken, except that its measure has been scaled by $p$. For the Sierpinsky triangle and a circularly symmetric wavelet, this is shown pictorially in Fig. 1.

Mathematically, this can be shown using the properties of the fractal object and of the analyzing wavelet. Specifically, from relation (5), we obtain

$$
W(a, \underline{b})=\sum_{i=1}^{N} p_{i} \int \psi\left(\frac{w_{i}(\underline{x})-\underline{b}}{a}\right) d \mu .
$$

Since $w_{i}(\underline{x})=\left(\lambda x+d_{1_{i}}, \lambda y+d_{2_{i}}\right)^{T}$, (8) can be rewritten as

$$
\begin{gathered}
\sum_{i=1}^{N} p_{i} \int \psi\left(\frac{x-\left(b_{1}-d_{1_{i}}\right) / \lambda}{a / \lambda}, \frac{y-\left(b_{2}-d_{2_{i}}\right) / \lambda}{a / \lambda}\right) d \mu \\
=\sum_{i=1}^{N} p_{i} W\left(a / \lambda, w_{i}^{-1}(\underline{b})\right) .
\end{gathered}
$$

We can choose the point $\underline{b}$ to be the lower left-most point in the support of the measure and translate the coordinate system such that $\underline{b}$ becomes the origin.

Definition (6) yields the identity

$$
\underline{y}-\underline{b}=Q_{i}(\underline{x}-\underline{b})+Q_{i} \underline{b}+\underline{d}_{i}-\underline{b} .
$$

In the new coordinate system, $\underline{y}^{\prime}=\underline{y}-\underline{b}$ and $\underline{x}^{\prime}=\underline{x}-\underline{b}$. Therefore, the IFS transformations become

$$
\underline{y}^{\prime}=Q_{i}^{\prime} \underline{x}^{\prime}+\underline{d}_{i}^{\prime}
$$

where

$$
Q_{i}^{\prime}=Q_{i}=\left[\begin{array}{cc}
\lambda_{x} & 0 \\
0 & \lambda_{y}
\end{array}\right] ; \quad \underline{d}^{\prime}=Q_{i} \underline{b}+\underline{d}_{i}-\underline{b}
$$

as it is seen immediately from $(10)$. Since $(0,0)$ is the lower left-most point in the support of the measure in the new coordinate system, there must be one transformation $w_{i_{0}}^{\prime}(\underline{x})$ that leaves the origin invariant, i.e., $w_{i_{0}}^{\prime}(0,0)=(0,0)$. Moreover, the transformations will have displacement vectors $\underline{d}_{i_{0}}^{\prime}=[0,0]^{T}$ and $\underline{d}_{j}^{\prime}=\left[d_{1_{j}}^{\prime}, d_{2_{j}}^{\prime}\right]^{T}$, where $d_{1_{j}}^{\prime}>0$ or $d_{2_{j}}^{\prime}>0$ for $j \neq i_{0}$ in the new coordinate system.

As a consequence, if the wavelet decays at infinity, only the term corresponding to $i_{0}$ contributes to the sum in (9) when the scale $a$ is sufficiently small. This is because all the other terms are obtained by centering the wavelet outside the attractor and therefore have a negligible contribution to the overall sum when the scale tends to 0 . Under these conditions, we have

$$
W(a, \underline{0}) \simeq p_{i_{0}} W\left(a / \lambda, w_{i_{0}}^{-1}(\underline{0})\right)=p_{i_{0}} W(a / \lambda, \underline{0}) .
$$

Relation (11) shows that the magnitude of the wavelet transform is multiplied by $1 / p_{i_{0}}$ every time we divide the scale by $\lambda$. This implies that the logarithm of the magnitude of the wavelet transform scales along the line

$$
\ln |W(\ln a, \underline{0})| \simeq \frac{\ln p_{i_{0}}}{\ln \lambda} \ln a+c
$$

and that for measures with nontrivial geometries, there are oscillations with period $a=1 / \lambda$ around the regression line. The reason for periodicity is that relation (12) is exact for any two values of $a$ that are $\lambda$ apart in logarithmic scale but approximate for any other values in between. Thus, the 
parameter $\lambda$ in (6) can be found by estimating the period of the difference between $\ln |W(\ln a, \underline{0})|$ and the least square regression line relating $\ln |W(\ln a, \underline{0})|$ to $\ln a$.

2) Case $\lambda_{x} \neq \lambda_{y}$ : A similar reasoning can be applied for the case $\lambda_{x} \neq \lambda_{y}$ in (6). We consider the two-scale wavelet transform

$$
W\left(a_{1}, a_{2} ; b_{1}, b_{2}\right)=\int \psi\left(\frac{x-b_{1}}{a_{1}}, \frac{y-b_{2}}{a_{2}}\right) d \mu .
$$

From (5), we get

$$
W\left(a_{1}, a_{2} ; \underline{b}\right)=\sum_{i=1}^{N} p_{i} W\left(\frac{a_{1}}{\lambda_{x}}, \frac{a_{2}}{\lambda_{y}} ; w_{i}^{-1}(\underline{b})\right) .
$$

Applying the same reasoning as above, and choosing $\underline{b}$ to be the lower left-most point in the support of the image and a new coordinate system such that $\underline{b} \equiv(0,0)$, we get

$$
W\left(a_{1}, a_{2} ; \underline{0}\right)=p_{i_{0}} W\left(\frac{a_{1}}{\lambda_{x}}, \frac{a_{2}}{\lambda_{y}} ; \underline{0}\right) .
$$

The logarithm of the two-scale wavelet transform therefore satisfies

$$
\begin{aligned}
\ln \left|W\left(\ln a_{1}, \ln a_{2} ; \underline{0}\right)\right| & \simeq \frac{\ln p_{i_{0}}}{\ln \lambda_{x}} \ln a_{1}+\frac{\ln p_{i_{0}}}{\ln \lambda_{y}} \ln a_{2}+c \\
& \triangleq T\left(\ln a_{1}, \ln a_{2}\right) .
\end{aligned}
$$

The difference

$$
r\left(\ln a_{1}, \ln a_{2}\right)=\ln \left|W\left(\ln a_{1}, \ln a_{2} ; \underline{0}\right)\right|-T\left(\ln a_{1}, \ln a_{2}\right)
$$

satisfies

$$
r\left(\ln a_{1}, \ln a_{2}\right)=r\left(\ln a_{1}-\ln \lambda_{x}, \ln a_{2}-\ln \lambda_{y}\right) .
$$

As a consequence, the Fourier transform $R\left(f_{x}, f_{y}\right)$ of $r\left(\ln a_{1}\right.$, $\ln a_{2}$ ) lies on the lines

$$
f_{y}=-\frac{\ln \lambda_{x}}{\ln \lambda_{y}} f_{x}+\frac{n_{1}}{\ln \lambda_{y}} \quad n_{1} \in \mathcal{Z} .
$$

The two parameters $\lambda_{x}$ and $\lambda_{y}$ can be therefore recovered by inspection of the Fourier transform $R\left(f_{x}, f_{y}\right)$ of the residue.

\section{B. Moment Method}

The general solution of the inverse problem requires the determination of the displacement parameters $d_{1_{i}}$ and $d_{2_{i}}$ and the number $N$ of the transformations; this is a problem that is considered partially in [8]. In this section, we propose a computational procedure for this problem based in part on the reasoning used in [9]-[11] for the 1-D case. Our approach is based on the fact that once $\lambda_{x}$ and $\lambda_{y}$ are known, it is possible to calculate from the image moments another set of moments of an atomic measure ${ }^{2}$ that are related to the displacement vectors and probabilities of the IFS that can therefore be computed from these moments using Padé approximation, as shown in Section III-B-1. In Section III-B-2, we discuss a way of finding the number of transformations.

\footnotetext{
${ }^{2} \mathrm{By}$ an atomic measure, we mean a measure corresponding to a discrete
} probability mass function.
1) Displacement Vectors and Probabilities: Substituting $f(x, y)=x^{h} y^{k}$ in (5), we show that the moments of the fractal measure, which are defined by

$$
\mu_{h, k}=\iint x^{h} y^{k} d \mu
$$

are related to the moments $\nu_{h, k}$

$$
\nu_{h, k}=\sum_{i=1}^{N} p_{i} d_{1_{i}}^{h} d_{2_{i}}^{k}
$$

of the atomic measure $\nu$

$$
d \nu=\sum_{i=1}^{N} p_{i} \delta\left(x-d_{1_{i}}\right) \delta\left(y-d_{2_{i}}\right) d x d y
$$

via the relationship

$$
\begin{gathered}
\nu_{h, k}=\mu_{h, k}\left(1-\lambda_{x}^{h} \lambda_{y}^{k}\right) \\
-\sum_{m} \sum_{n}\left(\begin{array}{c}
h \\
m
\end{array}\right)\left(\begin{array}{c}
k \\
n
\end{array}\right) \mu_{m, n} \lambda_{x}^{m} \lambda_{y}^{n} \nu_{h-m, k-n} \\
\nu_{0,0}=1, \\
(m, n) \in J=\{m, n \mid 0 \leq m \leq h, 0 \leq n \leq k, \\
(m, n) \neq(0,0),(m, n) \neq(h, k)\} .
\end{gathered}
$$

To see this, we have from (5)

$$
\begin{aligned}
\mu_{h, k}= & \int x^{h} y^{k} d \mu=\sum_{i=1}^{N} p_{i} \int\left(\lambda_{x} x+d_{1_{i}}\right)^{h}\left(\lambda_{y} y+d_{2_{i}}\right)^{k} d \mu \\
= & \sum_{i=1}^{N} p_{i} \int\left(\sum_{m=0}^{h}\left(\begin{array}{c}
h \\
m
\end{array}\right) \lambda_{x}^{m} x^{m} d_{1_{i}}^{h-m}\right) \\
& \times\left(\sum_{n=0}^{k}\left(\begin{array}{c}
k \\
n
\end{array}\right) \lambda_{y}^{n} y^{n} d_{2_{i}}^{k-n}\right) d \mu \\
= & \sum_{m=0}^{h} \sum_{n=0}^{k}\left(\begin{array}{c}
h \\
m
\end{array}\right)\left(\begin{array}{c}
k \\
n
\end{array}\right) \lambda_{x}^{m} \lambda_{y}^{n} \mu_{m, n} \nu_{h-m, k-n} .
\end{aligned}
$$

By taking the terms corresponding to $(m, n)=(0,0)$ and $(m, n)=(h, k)$ out of the summation and considering that $\mu_{0,0}=\nu_{0,0}=1$, we obtain (14).

Relation (14) shows that if an estimate of the moments $\mu_{h, k}$ is computed from the digital image of the fractal object, then $\nu_{h, k}$ can be evaluated using (14). Once $\nu_{h, k}$ is known, the problem of finding $d_{1_{i}}, d_{2_{i}}$, and $N$ can then be reduced to the problem of determining the unknown degree and system function of a 2-D discrete linear system characterized by a given 2-D impulse response array [18]. Indeed, taking the $\mathcal{Z}$ transform of $\nu_{h, k}$ in (13), we have

$$
\begin{aligned}
\mathcal{Z}\left\{\nu_{h, k}\right\} & \triangleq \sum_{h=0}^{\infty} \sum_{k=0}^{\infty} \nu_{h, k} z_{1}^{-h} z_{2}^{-k} \\
& =\sum_{i=1}^{N} p_{i} \frac{1}{1-z_{1}^{-1} d_{1_{i}}} \frac{1}{1-z_{2}^{-1} d_{2_{i}}} \\
& \triangleq \frac{A\left(z_{1}^{-1}, z_{2}^{-1}\right)}{B\left(z_{1}^{-1}, z_{2}^{-1}\right)}
\end{aligned}
$$


Letting $k=0$ and $h=0$ in (15), we obtain

$$
\begin{aligned}
& \sum_{h=0}^{\infty} \nu_{h, 0} z_{1}^{-h}=\sum_{i=1}^{N} p_{i} \frac{1}{1-z_{1}^{-1} d_{1_{i}}} \triangleq \frac{A_{1}\left(z_{1}^{-1}\right)}{B_{1}\left(z_{1}^{-1}\right)} \\
& \sum_{k=0}^{\infty} \nu_{0, k} z_{2}^{-k}=\sum_{i=1}^{N} p_{i} \frac{1}{1-z_{2}^{-1} d_{2_{i}}} \triangleq \frac{A_{2}\left(z_{2}^{-1}\right)}{B_{2}\left(z_{2}^{-1}\right)}
\end{aligned}
$$

where $B\left(z_{1}^{-1}, z_{2}^{-1}\right)=B_{1}\left(z_{1}^{-1}\right) B_{2}\left(z_{2}^{-1}\right)$. We use Padé approximation to obtain the coefficients of the polynomials in the denominators of (16) and (17) via solving a linear system of equations [18]. Consider the $N$-degree polynomial $B_{1}\left(z_{1}^{-1}\right)$ of (16)

$$
\begin{aligned}
B_{1}\left(z_{1}^{-1}\right) & \triangleq 1+b_{1} z_{1}^{-1}+\cdots+b_{N} z_{1}^{-N} \\
& =\sum_{i=0}^{N} b_{i} z_{1}^{-i}, \quad b_{0}=1 .
\end{aligned}
$$

Indeed, (16) constraints the convolution between the $N$ point sequence $b_{i}$ and the infinitely long sequence $\nu_{h, 0}$ to be 0 for the points $h=N, \ldots, 2 N-1$. This is because by definition, $A_{1}\left(z_{1}^{-1}\right)$ is a polynomial of degree $N-1$. Thus, the $b_{i}$ 's can be found by solving the linear system of equations

$$
\begin{gathered}
\left(b_{i} * \nu_{h, 0}\right)(k)=0, \quad k=N, \ldots, 2 N-1 \\
{\left[\begin{array}{cccc}
\nu_{0,0} & \nu_{1,0} & \ldots & \nu_{N-1,0} \\
\nu_{1,0} & \nu_{2,0} & \ldots & \nu_{N, 0} \\
\vdots & & \ldots & \vdots \\
\nu_{N-1,0} & \nu_{N, 0} & \ldots & \nu_{2 N-2,0}
\end{array}\right]\left[\begin{array}{c}
b_{N} \\
b_{N-1} \\
\vdots \\
b_{1}
\end{array}\right]=\left[\begin{array}{c}
-\nu_{N, 0} \\
-\nu_{N+1,0} \\
\vdots \\
-\nu_{2 N-1,0}
\end{array}\right]}
\end{gathered}
$$

where $*$ denotes convolution. Once $b_{i}$ 's are known, the roots of the polynomials $B_{1}\left(z_{1}^{-1}\right)$ and $B_{2}\left(z_{2}^{-1}\right)$ are used to find the displacement parameters $d_{1_{i}}$ and $d_{2_{i}}$, respectively. The nonzero coefficients of $A\left(z_{1}^{-1}, z_{2}^{-1}\right)$ in (15) are found by convolving the 2-D array $\nu_{h, k}$ with the identified 2-D denominator of (15), namely, $B\left(z_{1}^{-1}, z_{2}^{-1}\right) \triangleq B_{1}\left(z_{1}^{-1}\right) B_{2}\left(z_{2}^{-1}\right)$. Finally, from known $d_{1_{i}}$ and $d_{2_{i}}$, partial fraction expansion on $A\left(z_{1}^{-1}, z_{2}^{-1}\right) /$ $B\left(z_{1}^{-1}, z_{2}^{-1}\right)$ is used to determine the set of probabilities $p_{i}$.

2) Determining the Number of Transformations: One potential difficulty in the above procedure is that the number of transformations $N$ is not known in advance. Note that while $\underline{d}_{i}$ is different from $\underline{d}_{j}$ for $i \neq j$, it is possible to have $d_{1_{i}}=d_{1_{j}}$ (or equivalently $d_{2_{i}}=d_{2_{j}}$ ) for $i \neq j$. Therefore, the degree of the denominator polynomial $B_{1}\left(z_{1}^{-1}\right)$ in (16) (or equivalently $B_{2}\left(z_{2}^{-1}\right)$ in (17)) is not necessarily equal to the number $N$ of the transformations of the IFS. Rather, it corresponds to the number of distinct $d_{1_{i}}$ (or equivalently $d_{2_{i}}$ ).

Since the degrees $N_{1}$ and $N_{2}$ of the polynomials $B_{1}\left(z_{1}^{-1}\right)$ and $B_{2}\left(z_{2}^{-1}\right)$ are not known in advance, we consider a sequence of linear systems of equations of the kind in (18) corresponding to various polynomial degrees. Specifically, the assumed degree is incremented from one system to the next until the resulting Toeplitz system matrix becomes singular [12]. To see this, consider the case of a generic 1-D atomic measure $\nu$ with $N$-point support and moments

$$
\nu_{i}=\int x^{i} d \nu
$$

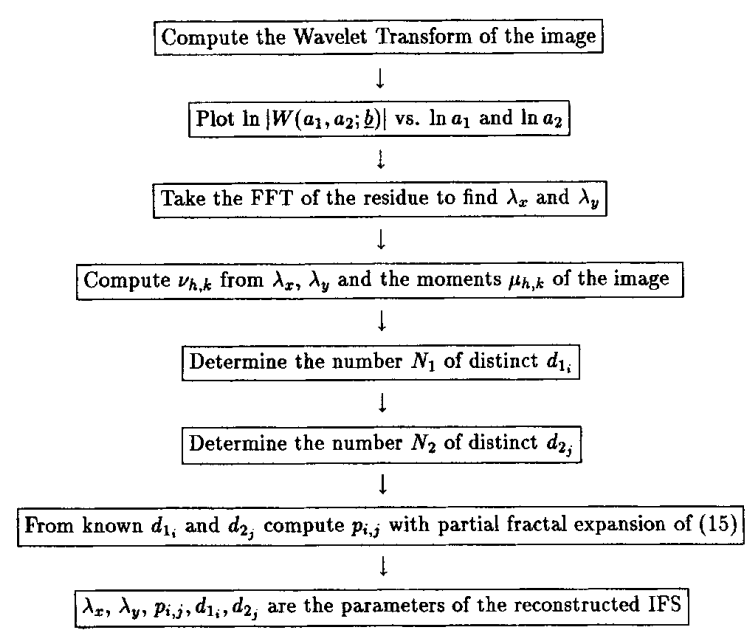

(a)

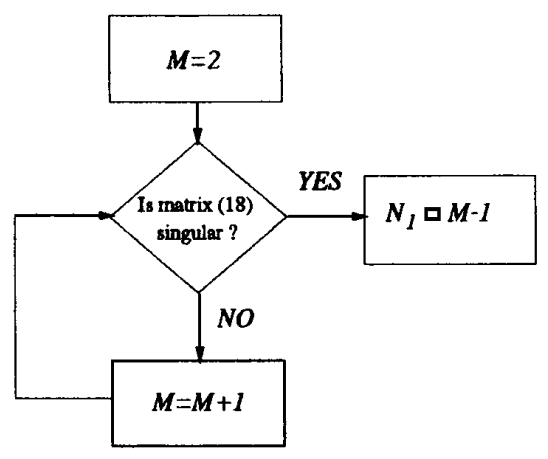

(b)

Fig. 2. (a) Flowchart for the inverse problem procedure; (b) determining $N_{1}$.

If we consider any polynomial $g(x)=\sum_{i=0}^{M} g_{i} x^{i}$ of degree $M<N$, the expectation with respect to the measure $\nu$ of $g^{2}(x)$ given by

$$
\begin{aligned}
E\left[g^{2}(x)\right] & =\sum_{i=0}^{M} \sum_{j=0}^{M} g_{i} g_{j} E\left[x^{i+j}\right] \\
& =\sum_{i=0}^{M} \sum_{j=0}^{M} g_{i} g_{j} \nu_{i+j}
\end{aligned}
$$

is a strictly positive number. This is because the expectation of a quantity that is always positive is also positive, and $g(x)$ has at most $M$ roots, whereas the measure is supported on $N>M$ points. This implies that the sequence of moments $\left\{\nu_{i}\right\}, i=0, \ldots, 2 M$ is positive definite, i.e.

$$
\sum_{i=0}^{M} \sum_{j=0}^{M} g_{i} g_{j} \nu_{i+j}>0, \quad \forall g_{i}, i=0, \ldots, M
$$




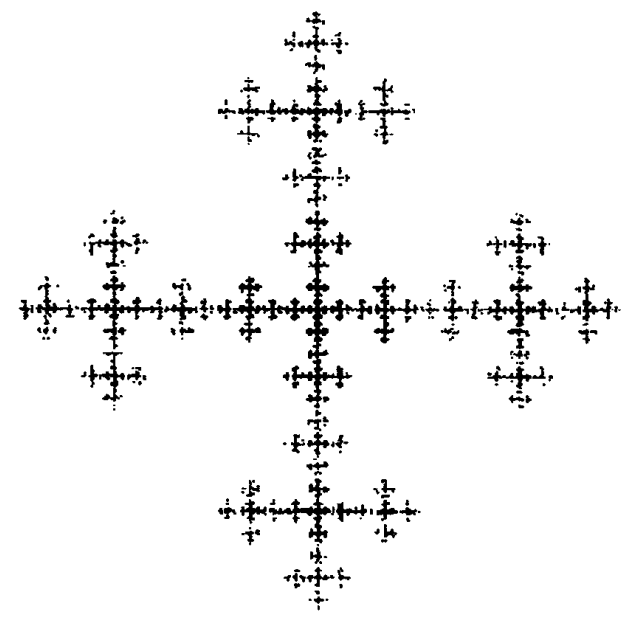

(a)

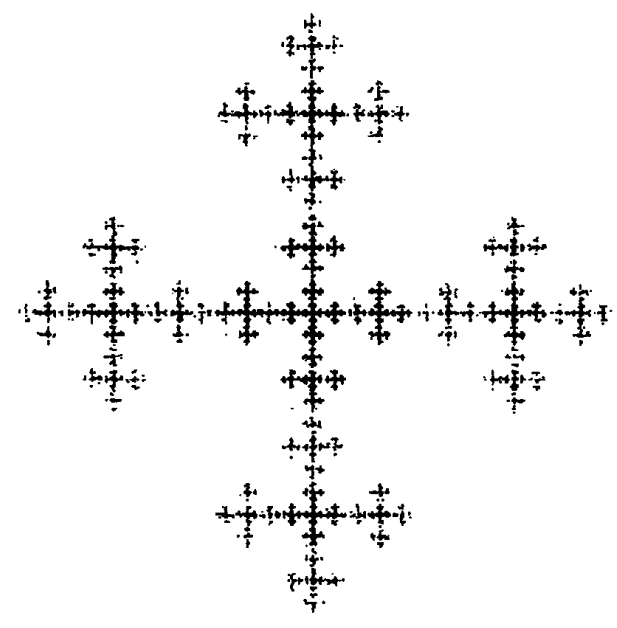

(b)

Fig. 3. Snowflake: (a) Original; (b) reconstructed.

or, equivalently, that the matrix

$$
\left[\begin{array}{cccc}
\nu_{0} & \nu_{1} & \ldots & \nu_{M} \\
\nu_{1} & \nu_{2} & \ldots & \nu_{M+1} \\
\vdots & & \ldots & \vdots \\
\nu_{M} & \nu_{M+1} & \ldots & \nu_{2 M}
\end{array}\right]
$$

is positive definite and has determinant greater than zero for $M<N$ [17]. For $M=N$ on the other hand, we can construct a polynomial $g(x)$ whose roots are in the same locations of the points of the support of the measure $\nu$, resulting in (19) to become zero and the corresponding matrix (20) to become positive semidefinite and singular.

Since the matrix in (20) is of the same same kind as the one in the left-hand side of (18) because $\nu_{h, 0}$ and $\nu_{0, k}$ are themselves the moments of a 1-D atomic measure, as seen from (13), our proposed procedure of incrementing the degree in (18) until it becomes singular will result in the determination of $N_{1}$ (or equivalently $N_{2}$ ). Specifically, as we argued above, the system in (18) is nonsingular for all $M<N_{1}$ and becomes singular as soon as $M=N_{1}$.

Once $N_{1}$ and $N_{2}$ are determined, displacement parameters and probabilities are determined using the technique described in Section III-B-1. Note that although $d_{1_{h}}$ and $d_{2_{k}}$ are known for $h=1, \ldots, N_{1}$ and $k=1, \ldots, N_{2}$, there is still ambiguity as to how they pair up. To resolve this, we find the residuals with respect to all possible pairs of $d_{1_{h}}$ and $d_{2_{k}}$ : in doing so, only the nonzero residuals correspond to $p_{i}$. The number of nonzero probabilities is equal to the number of transformations $N$ of the IFS.

A flow-chart summarizing the procedure for the inverse problem is shown in Fig. 2.

\section{RESUltS FOR THE INVERSE PROBLEM}

The procedure outlined above has been applied to the reconstruction of many IFS images obtained using the model of (6). The analyzing wavelet is the "Mexican hat" [6]

$$
\psi(x, y)=\left(2-x^{2}-y^{2}\right) \exp \left(-\frac{x^{2}+y^{2}}{2}\right) .
$$

As an illustration of the results, we consider the case of the Snowflake with nonequal probabilities. The Snowflake in Fig. 3(a) is obtained from an IFS with five affine transformations whose parameters are shown in Table I(a). While the images in Fig. 3(a) and (b) are rendered in this paper using the "chaos game," the actual inverse problem was solved on images generated via the Markov operator defined in Section II.

The displacement parameters and probabilities, along with the estimated ones, are listed in Table 1(a). As seen, the first five rows in Table 1(a) show the true and estimated displacement vectors and probabilities. The last four rows in Table 1(a) correspond to pairs of $d_{1_{i}}$ and $d_{2_{i}}$ that are not in the IFS under consideration. Thus, the corresponding probabilities are almost zero as expected. Fig. 4(a) plots the log-magnitude of the wavelet transform versus the log of the scale. As seen, periodicity is clearly noticeable in the residual, whose FFT is shown in Fig. 4(b). An estimate of the period gives $\lambda=0.330$, whereas the correct value is $\lambda=1 / 3$. Fig. 3(b) shows the reconstructed Snowflake. As seen, the reconstructed image looks indistinguishable from the original.

Fig. 5 shows the Sierpinsky triangle with probabilities, together with the reconstructed image. The IFS parameters and the estimated ones are in close agreement and are included in [22]. The log magnitude of the wavelet transform versus the $\log$ of the scale and the FFT of the residual are similar in nature to that of the Snowflake and are also included in [22]. 
TABLE I

IFS Parameters for the Snowflake (a) Original and Exact Reconstruction; (b) $(3,3)$ Order Approximation

\begin{tabular}{|c|c|c|c|c|c|}
\hline True $d_{1_{i}}$ & True $d_{2_{i}}$ & Est. $d_{1_{i}}$ & Est. $d_{2_{i}}$ & True $p_{i}$ & Est. $p_{i}$ \\
\hline $1 / 3$ & 0.0 & 0.332 & 0.000 & 0.15 & 0.150 \\
\hline 0.0 & $1 / 3$ & 0.000 & 0.332 & 0.15 & 0.150 \\
\hline $1 / 3$ & $1 / 3$ & 0.332 & 0.332 & 0.40 & 0.399 \\
\hline $1 / 3$ & $2 / 3$ & 0.332 & 0.666 & 0.15 & 0.150 \\
\hline \multirow[t]{5}{*}{$2 / 3$} & $1 / 3$ & 0.666 & 0.332 & 0.15 & 0.150 \\
\hline & & 0.000 & 0.000 & 0 & $1 E-5$ \\
\hline & & 0.000 & 0.666 & 0 & $6 E-6$ \\
\hline & & 0.666 & 0.000 & 0 & $6 E-6$ \\
\hline & & 0.666 & 0.666 & 0 & $1 E-5$ \\
\hline
\end{tabular}

(a)

\begin{tabular}{ccc}
\hline$d_{1_{i}}$ & $d_{2_{i}}$ & $p_{i}$ \\
\hline 0.375 & 0.375 & 0.403 \\
0.375 & 0.034 & 0.147 \\
0.375 & 0.716 & 0.147 \\
0.034 & 0.375 & 0.147 \\
0.034 & 0.034 & 0.002 \\
0.034 & 0.716 & 0.002 \\
0.716 & 0.375 & 0.147 \\
0.716 & 0.034 & 0.002 \\
0.716 & 0.716 & 0.002 \\
\hline \multicolumn{3}{c}{$\lambda_{x}=\lambda_{y}=0.25$}
\end{tabular}

(b)

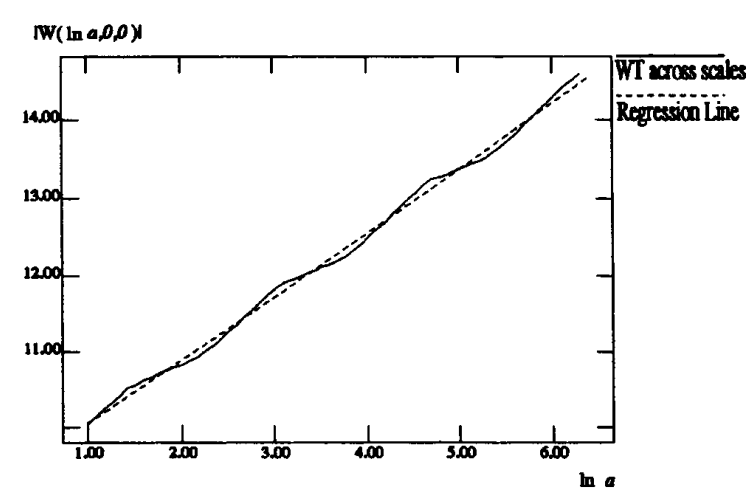

(a)

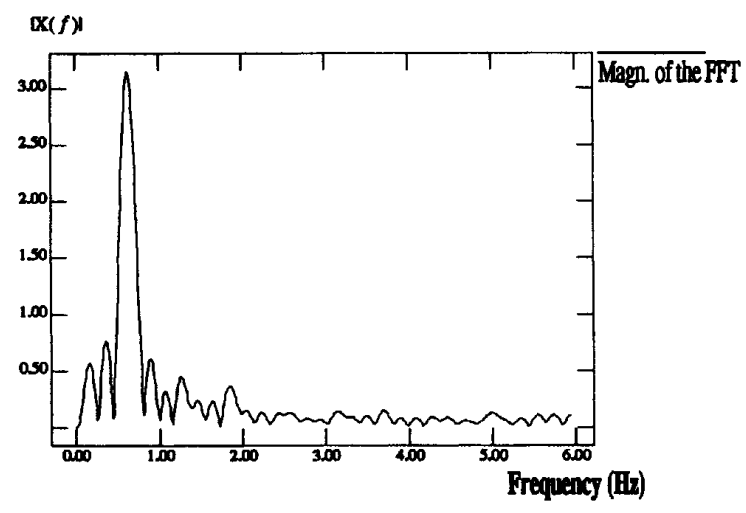

(b)

Fig. 4. Snowflake: (a) wavelet transform versus scale (log); (b) FFT of the residual.

We consider now the more general case of the overlapping IFS corresponding to the Fractal Butterfly of Fig. 6. In an overlapping IFS, the copies obtained by applying each transformation to the support of the attracting measure have nonzero superposition [1], [9]. Fig. 6 shows the original image and the reconstructed one after using the inverse procedure described above. The log-magnitude plot of the wavelet transform versus the $\log$ of the scale is similar to that of the Snowflake and is included in [22]. Moments $\mu_{h, k}$ and the wavelet transform were calculated from a $512 \times 512$ image obtained with the so called "chaos game" [1].

\section{APPROXIMATION PROCEDURES}

The procedure that was outlined in Section III is valid if the image was exactly generated with an IFS of type (6); this class of IFS is referred to as an homogeneous 2-D IFS because of the constant scaling factors $\lambda_{x}$ and $\lambda_{y}$. In that case, the properties of the fractal measure enabled us to use the wavelet transform to determine the scaling parameters of the IFS and solve the inverse problem exactly. In this section, we show that we can construct a homogeneous 2-D IFS whose invariant measure can be used in principle to approximate any given image, even though it is not generated via an IFS model. Since an arbitrary image does not possess in general any scale invariance, we cannot use the wavelet transform to determine the scaling parameters in our approximation procedures. The approximation is such that the 2-D moments $\tilde{\mu}_{h, k}$ of the IFS measure match the moments $\mu_{h, k}$ of the given image for a certain range $\mathcal{R}$ of indexes $(h, k)$. When successful, the suggested procedure always results in a valid IFS of the form (6).

Section V-A will present the approximation procedure with exact moment matching without any concern about the constraints that must be satisfied to make it valid. Validity issues will be analyzed in Section V-B. Another approximation scheme based on nonlinear optimization of the mean squared difference between the image moments and the IFS measure moments is considered in Section V-C. 


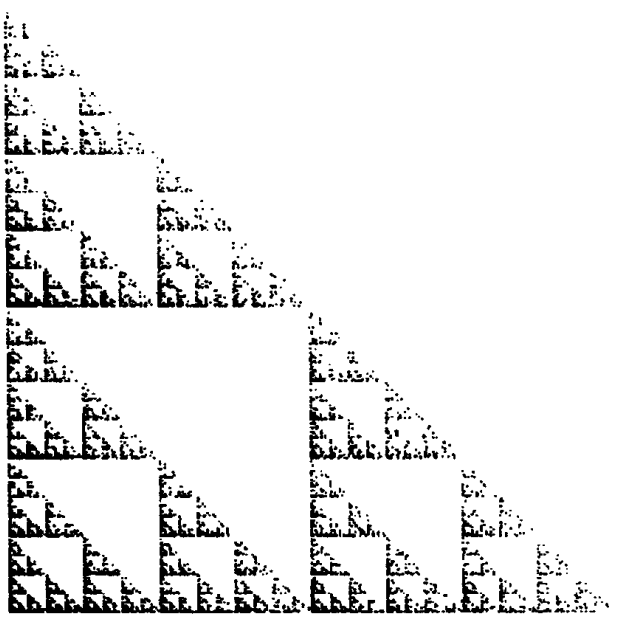

(a)

Fig. 5. Sierpinsky triangle with probabilities: (a) Original; (b) reconstructed.

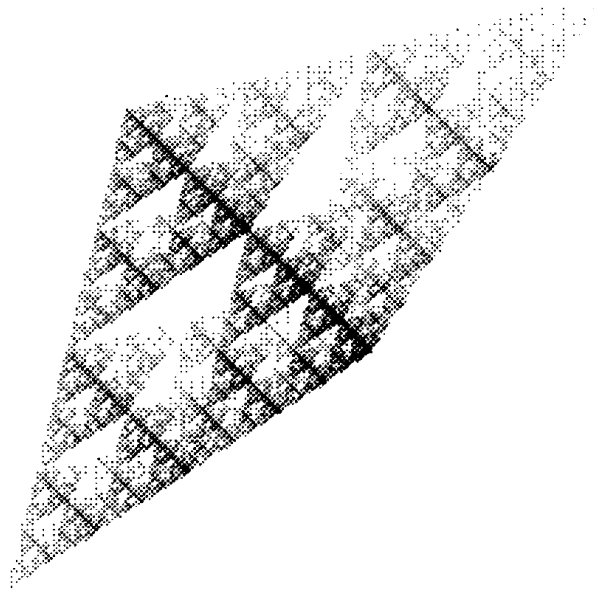

(a)

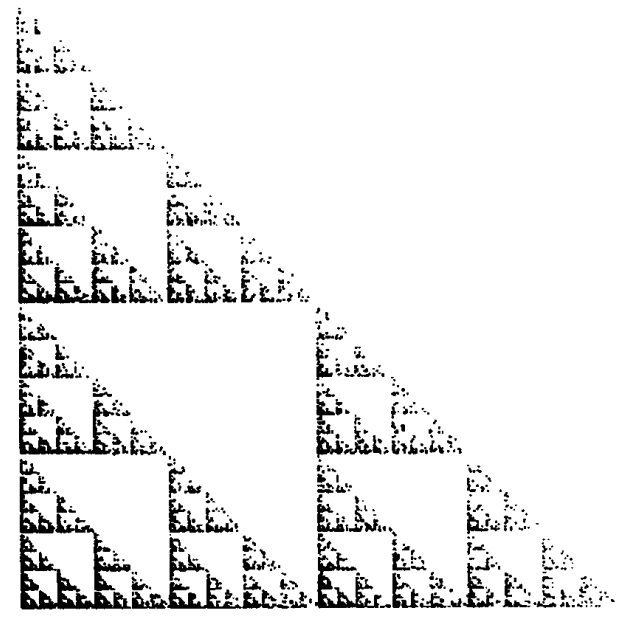

(b)

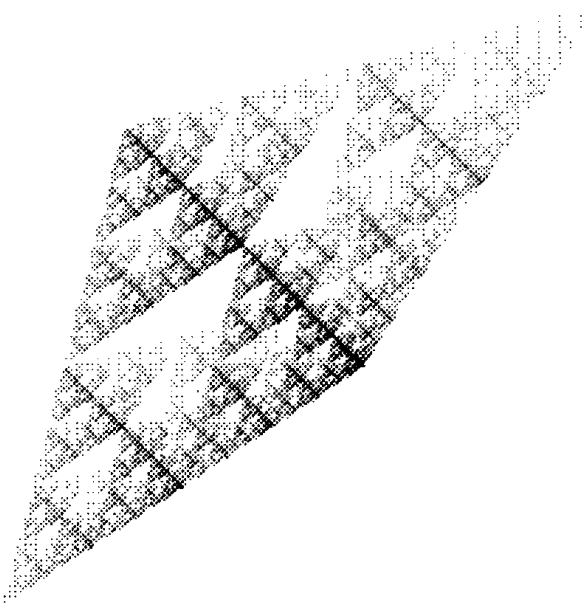

(b)

Fig. 6. Fractal Butterfly: (a) Original; (b) reconstructed.

\section{A. Approximation with Exact Moment Matching}

In this section, we will present the procedure to find an IFS measure whose moments $\tilde{\mu}_{h, k}$ exactly match a set of given moments $\mu_{h, k}$ calculated from an arbitrary image. Using a procedure similar to the one outlined in Section III, we find a set of numbers $\nu_{h, k}$ from which we calculate the parameters of the IFS system. Contrary to the case of the inverse problem, where the original image is exactly generated via an IFS and the wavelet transform can be used to find the scaling parameters, in approximating arbitrary non-IFSgenerated images, there is freedom in the choice of the scaling parameters $\lambda_{x}$ and $\lambda_{y}$ even when we fix the number of transformations of the IFS. Therefore, the approximation is not uniquely determined.

Our approach to this problem is as follows. First, we show in this section that for any scaling parameters $\lambda_{x}$ and $\lambda_{y}$, the displacement vectors and probabilities can be computed. In Section V-B, we show constraints on $\lambda_{x}$ and $\lambda_{y}$ such that the resulting probabilities are positive, and the displacement parameters are real numbers.

Let us assume that a finite number of moments $\mu_{h, k}$ of our target measure $\mu$ are available. Without loss of generality, the support of the measure $\mu$ will be considered in the following to be included in the square $[0,1) \times[0,1)$. If $\mu$ is in the form of a digitized image, the moments $\mu_{h, k}$, which are defined by 


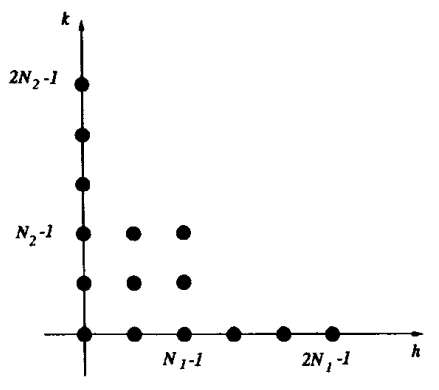

Fig. 7. Region $\mathcal{R}$ for a $N_{1}=3, N_{2}=3$ IFS approximation.

the integral

$$
\mu_{h, k}=\int x^{h} y^{k} d \mu
$$

can be readily computed since the measure $\mu$ has a density that is piecewise constant over each pixel of the image.

Our goal is to find, when possible, an IFS measure $\tilde{\mu}$ whose moments $\tilde{\mu}_{h, k}$ are equal to those of $\mu$ in a region $\mathcal{R}$ of indexes $(h, k)$. The size of the region $\mathcal{R}$ depends on the number of transformations that we choose for the approximating IFS; if the IFS of type (6) has $N_{1}$ different displacement parameters $d_{1_{i}}, i=1, \ldots, N_{1}$, and $N_{2}$ different displacement parameters $d_{2_{j}}, j=1, \ldots, N_{2}$, then the IFS will consist of $N_{1} N_{2}$ transformations, where each corresponds to a pair $\left(d_{1_{i}}, d_{2_{j}}\right)$

$$
\begin{aligned}
w_{i, j}(\underline{x})= & {\left[\begin{array}{cc}
\lambda_{x} & 0 \\
0 & \lambda_{y}
\end{array}\right]\left[\begin{array}{l}
x \\
y
\end{array}\right]+\left[\begin{array}{l}
d_{1_{i}} \\
d_{2_{j}}
\end{array}\right] ; } \\
& i=1, \ldots, N_{1} ; \quad j=1, \ldots, N_{2}
\end{aligned}
$$

and probability $p_{i, j}$. We will denote by $\left(N_{1}, N_{2}\right)$ the order of the approximation. The region $\mathcal{R}$ for a $N_{1}=3, N_{2}=3$ approximation is shown in Fig. 7. The particular shape of region $\mathcal{R}$ is due to the class of IFS (22) that we consider, as we will show below in the description of the approximation procedure.

Given a finite set of moments $\mu_{h, k}$, we can generate, for any arbitrary fixed value of $\lambda_{x}$ and $\lambda_{y}$, a sequence $\nu_{h, k}$ of numbers using relation (14). We then search for a 2-D atomic measure

$$
d \tilde{\nu}=\sum_{i=1}^{N_{1}} \sum_{j=1}^{N_{2}} p_{i, j} \delta\left(x-d_{1_{i}}\right) \delta\left(y-d_{2_{j}}\right) d x d y
$$

whose moments

$$
\tilde{\nu}_{h, k}=\sum_{i=1}^{N_{1}} \sum_{j=1}^{N_{2}} p_{i, j} d_{1_{i}}^{h} d_{2_{j}}^{k}
$$

match $\nu_{h, k}$ in $\mathcal{R}$, i.e., $\nu_{h, k}=\tilde{\nu}_{h, k}$ for $(h, k) \in \mathcal{R}$. We have from (23)

$$
\begin{aligned}
\mathcal{Z}\left\{\tilde{\nu}_{h, k}\right\} & =\sum_{i=1}^{N_{1}} \sum_{j=1}^{N_{2}} \frac{p_{i, j}}{\left(1-d_{1_{i}} z_{1}^{-1}\right)\left(1-d_{2_{j}} z_{2}^{-1}\right)} \\
& \triangleq \frac{A\left(z_{1}^{-1}, z_{2}^{-1}\right)}{B\left(z_{1}^{-1}, z_{2}^{-1}\right)} .
\end{aligned}
$$

The procedure to find the parameters $p_{i, j}$ and $d_{1_{i}}, d_{2_{j}}$ is now similar to the one outlined in Section III for the exact inverse problem and is based on 2-D Padé approximation. Specifically, from (23), we have

$$
\sum_{h=0}^{\infty} \tilde{\nu}_{h, 0} z_{1}^{-h}=\sum_{i=1}^{N_{1}}\left(\frac{\sum_{j=1}^{N_{2}} p_{i, j}}{1-d_{1_{i}} z_{1}^{-1}}\right) \triangleq \frac{A_{1}\left(z_{1}^{-1}\right)}{B_{1}\left(z_{1}^{-1}\right)}
$$

where $\operatorname{deg} B_{1}\left(z_{1}\right)=N_{1}$ and $\operatorname{deg} A_{1}\left(z_{1}\right)=N_{1}-1$. If $A_{1}\left(z_{1}^{-1}\right) / B_{1}\left(z_{1}^{-1}\right)$ is computed as the $\left[N_{1}-1 / N_{1}\right]$ Padé approximation [18] of the sequence $\left\{\nu_{h, 0}\right\}$, i.e.

$$
\sum_{h=0}^{\infty} \nu_{h, 0} z_{1}^{-h}=\frac{A_{1}\left(z_{1}^{-1}\right)}{B_{1}\left(z_{1}^{-1}\right)}+\mathcal{O}\left(z_{1}^{-2 N_{1}}\right)
$$

then we can set $\tilde{\nu}_{h, 0}=\nu_{h, 0}$ for $h=0,1, \ldots, 2 N_{1}-1$. The Padé approximation $A_{1}\left(z_{1}^{-1}\right) / B_{1}\left(z_{1}^{-1}\right)$ is readily computed by solving a linear system of equations and computing convolution, as shown in Section III [18].

In a similar way, we can find polynomials $A_{2}\left(z_{2}^{-1}\right) / B_{2}\left(z_{2}^{-1}\right)$ such that

$$
\begin{aligned}
& \frac{A_{2}\left(z_{2}^{-1}\right)}{B_{2}\left(z_{2}^{-1}\right)} \triangleq \sum_{k=0}^{\infty} \tilde{\nu}_{0, k} z_{2}^{-k}, \\
& \sum_{k=0}^{\infty} \nu_{0, k} z_{2}^{-k}=\frac{A_{2}\left(z_{2}^{-1}\right)}{B_{2}\left(z_{2}^{-1}\right)}+\mathcal{O}\left(z_{2}^{-2 N_{2}}\right) ; \\
& \nu_{0, k}=\tilde{\nu}_{0, k}, \quad k=0,1, \ldots, 2 N_{2}-1 .
\end{aligned}
$$

We note that because of relation (25), the displacement parameters $d_{1_{i}}, i=1, \ldots, N_{1}$ can be found as the roots of the polynomial $B_{1}\left(z_{1}^{-1}\right)$. Similarly, $d_{2_{j}}, j=1, \ldots, N_{2}$ are the roots of the polynomial $B_{2}\left(z_{2}^{-1}\right)$.

Once $B_{1}\left(z_{1}^{-1}\right)$ and $B_{2}\left(z_{2}^{-1}\right)$ are computed, the coefficients of $A\left(z_{1}^{-1}, z_{2}^{-1}\right)$ in (24) are found by computing the 2-D convolution of $\nu_{h, k}$ with the separable sequence $B\left(z_{1}^{-1}, z_{2}^{-1}\right)$ $\triangleq B_{1}\left(z_{1}^{-1}\right) B_{2}\left(z_{2}^{-1}\right)$

$\left(\nu_{i, j} * *(b)\right)(h, k), \quad h=0, \ldots, N_{1}-1 ; \quad k=0, \ldots, N_{2}-1$.

Finally, probability $p_{i, j}$ is determined by finding the residual of (24) with respect to the pair of roots $d_{1_{i}}$ and $d_{2_{j}}$.

The above procedure guarantees that the 2-D rational function

$$
\frac{A\left(z_{1}^{-1}, z_{2}^{-1}\right)}{B\left(z_{1}^{-1}, z_{2}^{-1}\right)}
$$

has inverse $\mathcal{Z}$-transform coefficients

$$
\begin{aligned}
\tilde{\nu}_{h, k}= & \sum_{i=1}^{N_{1}} \sum_{j=1}^{N_{2}} p_{i, j} d_{1_{i}}^{h} d_{2_{j}}^{k}=\nu_{h, k} \\
(h, k) \in \mathcal{R}= & \left\{0 \leq h \leq N_{1}-1,0 \leq k \leq N_{2}-1\right\} \\
& \cup\left\{h=0, N_{2} \leq k \leq 2 N_{2}-1\right\} \\
& \cup\left\{N_{1} \leq h \leq 2 N_{1}-1, k=0\right\} .
\end{aligned}
$$

The region $\mathcal{R}$ in (29) is explained by considering that the 1$D$ Padé approximation of (26) guarantees the equivalence of moments for $0 \leq h \leq 2 N_{1}-1$, the 1-D Padé approximation 


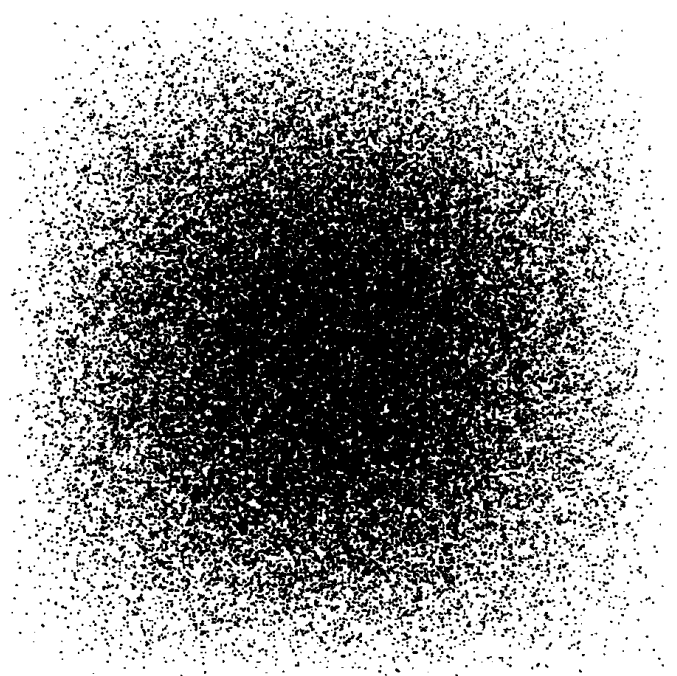

Fig. 8. $(2,2)$ order approximation of the Snowflake.

of (27) guarantees the equivalence of moments for $0 \leq k \leq$ $2 N_{2}-1$, and the 2 -D convolution of (28) guarantees the equivalence for $0<h \leq N_{1}-1,0<k \leq N_{2}-1$. This explains the shape of the matching region in Fig. 7. The IFS of type (6) corresponding to the parameters $p_{i, j}, d_{1_{i}}, d_{2_{j}}$ and the chosen $\lambda_{x}, \lambda_{y}$ will have moments $\tilde{\mu}_{h, k}$ that can be calculated from $\tilde{\nu}_{h, k}$ by inverting relation (14), namely

$$
\begin{aligned}
\tilde{\mu}_{0,0} & =1 ; \\
\tilde{\mu}_{h, k} & =\left(1-\lambda_{x}^{h} \lambda_{y}^{k}\right)^{-1} \\
& \cdot\left[\sum_{\substack{m=0 \\
(m, n) \neq(h, k)}}^{h} \sum_{n=0}^{k}\left(\begin{array}{c}
h \\
m
\end{array}\right)\left(\begin{array}{l}
k \\
n
\end{array}\right) \tilde{\mu}_{m, n} \lambda_{x}^{m} \lambda_{y}^{n} \tilde{\nu}_{h-m, k-n}\right] .
\end{aligned}
$$

Relations (30) and (14) show that there is a one-to-one correspondence between $\tilde{\mu}_{h, k}$ and $\tilde{\nu}_{h, k}$ for $(h, k) \in \mathcal{R}$. The fact $\tilde{\nu}_{h, k}=\nu_{h, k}$ for $(h, k) \in \mathcal{R}$ therefore guarantees that the attracting measure $\tilde{\mu}$ of the IFS thus determined has the desired original moments $\tilde{\mu}_{h, k}=\mu_{h, k}$ for $(h, k) \in \mathcal{R}$.

\section{B. Validity of the Approximation}

The construction above is consistent only if the resulting IFS is a valid one. By a valid IFS, we mean one where probabilities $p_{i, j}$ are positive and displacement parameters $d_{1}$ and $d_{2_{j}}$ are real numbers. The choice of the parameters $\lambda_{x}$ and $\lambda_{y}$ provides the required degree of freedom to search for a valid approximation. In this section, we will present the procedure we adopt to obtain a valid approximating IFS, and the constraints the IFS parameters must obey. In Section V-B-1, we discuss conditions under which the displacement parameters result to be real, and in Section V-B-2, we discuss conditions under which the probabilities are positive.
1) Realness of Displacement Parameters: The theoretical framework that we consider is the so-called "problem of moments" [12]. In particular, we reproduce here the following lemma from [12] and [9]:

Lemma 1: Given a set of numbers $\left\{\mu_{0}, \mu_{1}, \ldots, \mu_{2 L-1}\right\}$, consider the Hankel-Hadamard determinants $\Delta_{n}^{(j)}, j=0,1,2$ of the $(n+1) \times(n+1)$ real symmetric matrices $H_{n}^{(j)}$ with entries

$$
\begin{aligned}
H_{n}^{(j)}(h, k) & =\mu_{j+h+k} \\
j=0,1, \quad(h, k), h & =0, \ldots, n ; \quad k=0, \ldots, n
\end{aligned}
$$

and

$$
\begin{aligned}
H_{n}^{(2)}(h, k) & =\mu_{h+k}-\mu_{h+k+1}, \quad(h, k), \\
h & =0, \ldots, n ; \quad k=0, \ldots, n .
\end{aligned}
$$

If the inequalities

$$
\Delta_{n}^{(j)}>0, \quad j=0,1,2 ; \quad n=0,1, \ldots, L-1
$$

are satisfied, then there exists an $L$-point atomic measure

$$
d \nu=\sum_{n=1}^{L} p_{n} \delta\left(x-d_{n}\right)
$$

with support in $[0,1]$ having $\left\{\mu_{0}, \mu_{1}, \ldots, \mu_{2 L-1}\right\}$ as moments. Moreover, the locations $d_{n}$ and weights $p_{n}$ can be determined from the $[L-1 / L]$ Padé approximant

$$
\begin{aligned}
{[L-1 / L]\left(z^{-1}\right) } & =\sum_{n=1}^{L} \frac{p_{n}}{1-d_{n} z^{-1}} \\
& =\sum_{n=0}^{2 L-1} \mu_{n} z^{-n}+\mathcal{O}\left(z^{-2 L}\right)
\end{aligned}
$$

where the weights $p_{n}$ 's are guaranteed to be nonnegative, and $d_{n}$ 's are guaranteed to be real numbers. Conversely, the set of moments $\left\{\mu_{0}, \mu_{1}, \ldots, \mu_{2 L-1}\right\}$ of any positive measure verifies (33).

If we do not require that the support of the measure is $[0$, 1], then condition (33) can be relaxed to

$$
\Delta_{n}^{(0)}>0, \quad n=0,1, \ldots, L-1 .
$$

This still guarantees that the $d_{n}$ 's are real numbers but not necessarily between 0 and 1 .

The previous lemma suggests that if we choose the scaling parameters $\lambda_{x}$ and $\lambda_{y}$ in our approximating procedure such that conditions in (33) are satisfied for the sequences $\left\{\nu_{h, 0}\right\}$, $h=0, \ldots, 2 N_{1}-1$ and $\left\{\nu_{0, k}\right\}, k=0, \ldots, 2 N_{2}-1$ obtained from the moments $\mu_{h, k}$ via (14), then the computed displacement parameters $d_{1_{i}}$ and $d_{2_{j}}$ will be indeed real numbers. One way to satisfy (33) is to consider the case where $\lambda_{x}=\lambda_{y}=0$. Under these conditions, we have from (14) $\nu_{h, 0}=\mu_{h, 0}$ and $\nu_{0, k}=\mu_{0, k}$. Sequences $\mu_{h, 0}$ and $\mu_{0, k}$ are obtained from the 1-D marginal distributions of the 2-D measure $\mu$ and are therefore the moments of a 1-D measure. As a consequence, they satisfy the conditions of (33). Since the constraints (33) are continuous in $\lambda_{x}$ and $\lambda_{y}$, there exists 


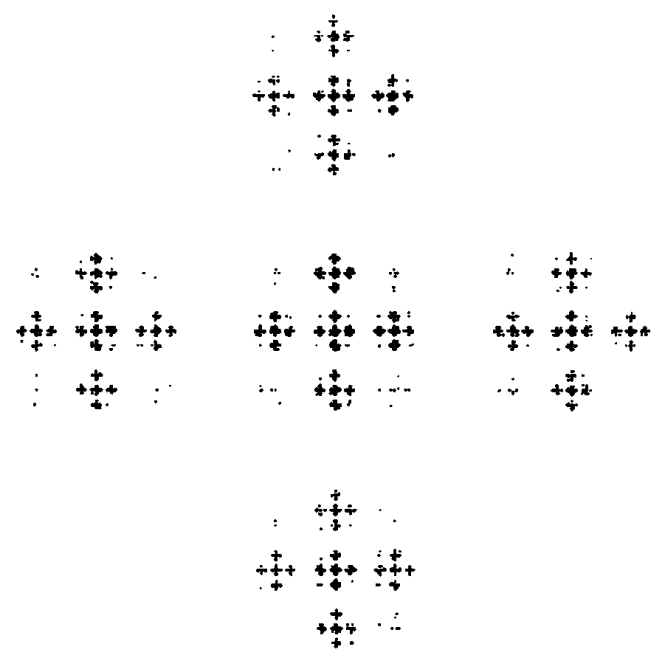

(a)

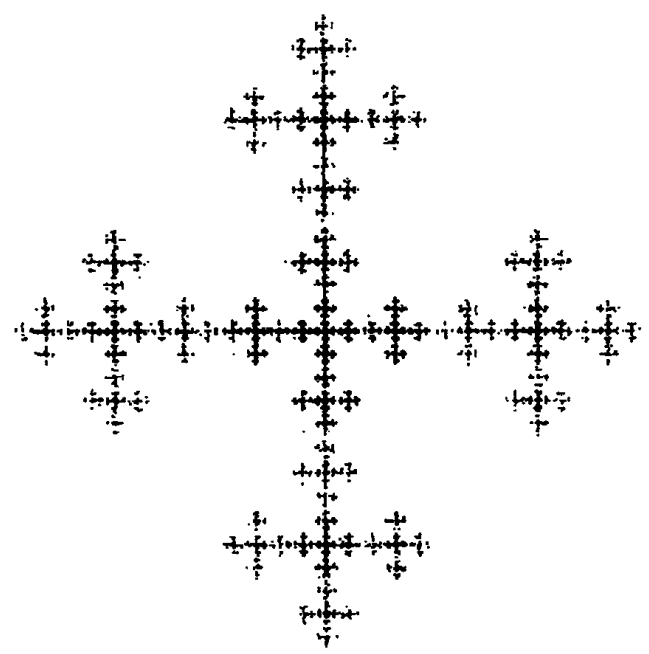

(b)

Fig. 9. (3, 3) order approximation of the Snowflake: (a) $\lambda_{x}=\lambda_{y}=0.25$; (b) $\lambda_{x}=\lambda_{y}=0.33$.

a range $I_{x}=\left[0, \lambda_{x_{\max }}\right]$ for $\lambda_{x}$ and a range $I_{y}=\left[0, \lambda_{y_{\max }}\right]$ for $\lambda_{y}$ such that $\nu_{h, 0}$ and $\nu_{0, k}$ satisfy (33).

We can summarize the above discussion in the following theorem:

Theorem 1: Given the moments $\left\{\mu_{h, k}\right\}$ of the original measure $\mu$, it is possible to find an interval $I_{x}=\left[0, \lambda_{x_{\max }}\right]$ of values of $\lambda_{x}$ and $I_{y}=\left[0, \lambda_{y_{\max }}\right]$ for $\lambda_{y}$ such that the moments $\left\{\nu_{h, k}\right\}$ calculated from $\left\{\mu_{h, k}\right\}$ via (14) will result in real numbers $d_{1_{i}}$ and $d_{2_{j}}$ when using the approximation procedure of Section V-A.

Values of $\lambda_{x}$ or $\lambda_{y}$ obtained in the above theorem is one way to satisfy conditions (33) but certainly not the only way. In general, the situation is more complex in that there exists more than one interval of values of $\lambda_{x}$ (or $\lambda_{y}$ ) such that conditions (33) are satisfied for the corresponding sequence $\nu_{h, 0}$ (or $\nu_{0, k}$ ). This observation is quite important; even if we attempt to approximate an image that has been obtained exactly with an IFS of the form (6) and even if we use a number of transformations that exceeds the one used in generating the original image, we may fail to recover the IFS exactly if we confine the search of the parameter $\lambda_{x}$ (or $\lambda_{y}$ ) to the interval $I_{x}$ (or $I_{y}$ ).

Besides the intervals $I_{x}$ and $I_{y}$ of Theorem 1, there will be other intervals of values of $\lambda_{x}$ (or $\lambda_{y}$ ) in which conditions (33) are satisfied. Let us denote by $U_{x}$ (or $U_{y}$ ) the union of such intervals. A general way of determining $U_{x}$ (or $U_{y}$ ) is to check the smallest eigenvalue of the matrices $H_{N_{1}-1}^{(j)}$ for the sequence $\nu_{h, 0}$ (or $H_{N_{2}-1}^{(j)}$ for the sequence $\nu_{0, k}$ ) as a function of $\lambda_{x}$ (or $\lambda_{y}$ ). The regions in which such eigenvalue is greater than 0 correspond to a positive definite $H_{N_{1}-1}^{(j)}$ so that conditions in (33) are satisfied [17].

2) Positivity of Probabilities; Thus far, we have dealt with choosing $\lambda_{x}$ and $\lambda_{y}$ so that the displacement parameters are real and in the range $[0,1]$. Another constraint that needs to be satisfied to assure the validity of our approximation procedure is the positivity of the $p_{i, j}$. Note that Lemma 1 does not guarantee the positivity of probabilities $p_{i, j}$ because it only deals with 1-D atomic approximations. As for the positivity of the probabilities $p_{i, j}$. we have the following result.

Theorem 2: The 2-D homogeneous IFS approximation procedure of order $\left(N_{1}, N_{2}\right)$ will result in positive probabilities if and only if there exist $\lambda_{x} \in U_{x}$ and $\lambda_{y} \in U_{y}$ such that the polynomials

$$
\begin{aligned}
t_{h, k ; \lambda_{x}, \lambda_{y}}(x, y)= & \left(\prod_{\substack{i=1 \\
i \neq h}}^{N_{1}} \frac{x-d_{1_{i}}}{d_{1_{h}}-d_{1_{i}}}\right)\left(\prod_{\substack{j=1 \\
j \neq k}}^{N_{2}} \frac{y-d_{2_{j}}}{d_{2_{k}}-d_{2_{j}}}\right) \\
\triangleq & \sum_{i=0}^{N_{1}-1} \sum_{j=0}^{N_{2}-1} a_{h, k ; i, j} x^{i} y^{j} \\
& h=1, \ldots, N_{1} ; \quad k=1, \ldots, N_{2}
\end{aligned}
$$

satisfy

$\sum_{i=0}^{N_{1}-1} \sum_{j=0}^{N_{2}-1} a_{h, k ; i, j} \nu_{i, j} \geq 0, h=1, \ldots, N_{1} ; k=1, \ldots, N_{2}$.

Proof: The polynomial $t_{h, k ; \lambda_{x}, \lambda_{y}}(x, y)$ is the unique polynomial of degree $N_{1}-1$ in $x$ and $N_{2}-1$ in $y$ satisfying

$$
t_{h, k ; \lambda_{x}, \lambda_{y}}\left(d_{1_{i}}, d_{2_{j}}\right)= \begin{cases}1 & i=h, j=k \\ 0 & \text { otherwise }\end{cases}
$$

Given a not-necessarily positive atomic measure $\tilde{\nu}$ with weights ${ }^{3} p_{h, k}$ at the mass points $\left(d_{1_{h}}, d_{2_{k}}\right)$ and moments

\footnotetext{
${ }^{3}$ We reserve the name probabilities for positive weights $p_{h, k}$.
} 


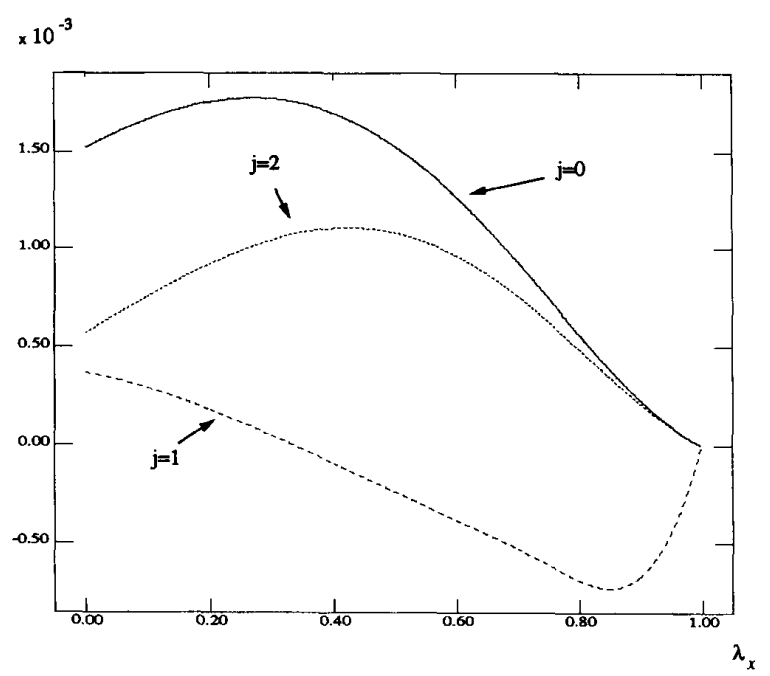

Fig. 10. Smallest eigenvalue of $H_{2}^{(j)}, j=0,1,2$ versus $\lambda_{x}$ for a $(3,3)$ approximation.

$\tilde{\nu}_{h, k}=\nu_{h, k}$ for $(h, k) \in \mathcal{R}$, we have

$$
p_{h, k}=E\left[t_{h, k ; \lambda_{x}, \lambda_{y}}(x, y)\right]=\sum_{i=0}^{N_{1}-1} \sum_{j=0}^{N_{2}-1} a_{h, k ; i, j} \nu_{i, j}
$$

where $E[\cdot]$ denotes the expectation operator with respect to the measure $\tilde{\nu}$. This is because the polynomial $t_{h, k ; \lambda_{x}, \lambda_{y}}(x, y)$ is zero at all the mass points of the measure except at the one with weight $p_{h, k}$. Therefore, $p_{h, k} \geq 0$, for $h=1, \ldots, N_{1} ; k=$ $1, \ldots, N_{2}$ if and only if there are $\lambda_{x}$ and $\lambda_{y}$ such that (35) is satisfied.

Thus, to ensure positivity of the probabilities, we must ensure that $\lambda_{x}$ and $\lambda_{y}$ are chosen so that (35) is satisfied.

From Theorems 1 and 2 and the results of Section V-A, the complete approximation procedure with an IFS of order $\left(N_{1}, N_{2}\right)$ can be outlined as follows:

1) Determine ranges $U_{x}$ and $U_{y}$ such that the sequence $\left\{\nu_{h, 0}\right\}$ computed from $\mu_{h, k}$ via (14) satisfies

$$
\Delta_{m}^{(j)}>0, \quad j=0,1,2, \quad m=0, \ldots, N_{1}-1
$$

and the sequence $\left\{\nu_{0, k}\right\}$ satisfies

$$
\Delta_{n}^{(j)}>0, \quad j=0,1,2, \quad n=0, \ldots, N_{2}-1 .
$$

2) Choose a value of $\lambda_{x}$ in $U_{x}$ and $\lambda_{y}$ in $U_{y}$, calculate $d_{1_{i}}$ and $d_{2 j}$ using 1-D Padé approximation of the sequences $\left\{\nu_{h, 0}\right\}, h=0, \ldots, 2 N_{1}-1$, and $\left\{\nu_{0, k}\right\}, k=$ $0, \ldots, 2 N_{2}-1$.

3) Build the polynomials $t_{h, k ; \lambda_{x}, \lambda_{y}}(x, y)$ of Theorem 2 for $h=1, \ldots, N_{1}, k=1, \ldots, N_{2}$, and check conditions in (35)

4) If the conditions are met for the chosen $\lambda_{x}$ and $\lambda_{y}$ or if the direct check of the probabilities indicates that they are all positive, then the approximation procedure is successful.
5) Otherwise, choose a different value of $\lambda_{x}$ in $U_{x}$ and $\lambda_{y}$ in $U_{y}$, and continue from step 2 .

Note that the approximation procedure is not unique. In particular, any value of $\lambda_{x}$ and $\lambda_{y}$ in the allowable ranges $U_{x}$ and $U_{y}$ that results in positive probabilities provides a solution of the exact matching moment procedure. We will see the implications of this in the experimental results of Section VI.

\section{Approximation with Overdetermined Moment Matching}

In the last section, we showed a procedure to determine an IFS whose attractive measure has exactly the same moments of the original given image in the range $\mathcal{R}$. In this section, we consider a procedure aimed at finding an IFS measure whose moments approximate the given ones in a mean squared sense.

Equation (30) shows that the moments $\tilde{\mu}_{h, k}$ of the IFS approximating measure are a nonlinear function of the parameters $P=\left\{\lambda_{x}, \lambda_{y} ; p_{i, j}, d_{1_{i}}, d_{2_{j}}, i=1, \ldots, N_{1}, j=1, \ldots\right.$, $\left.N_{2}\right\}$. It is therefore conceivable to find the approximating IFS by calculating the parameters that minimize the objective function

$$
O_{\mu}=\sum_{h, k}\left(\mu_{h, k}-\tilde{\mu}_{h, k}\right)^{2}
$$

over a certain region of indexes $\mathcal{R}$. In [16], the same method is applied to the more complex case of a general matrix $Q_{i}$ in (2). As we will see in Section VI, the main difference between our approach and that of [16] is that we can use the parameters obtained in the exact moment matching as described in Section $\mathrm{V}-\mathrm{A}$ as the initial conditions in the nonlinear approximation problem.

In our minimization procedure, the parameters must satisfy a set of constraints that make the resulting IFS a valid one, namely, the parameters $\lambda_{x}$ and $\lambda_{y}$ must be positive numbers less than 1 to make the IFS transformations contractive, $p_{i, j}$ must be a set of positive probabilities, and $d_{1_{i}}, d_{2_{j}}$ must be in $[0,1]$ to have a resulting fractal measure with compact support. The approximation problem therefore becomes a linearly constrained nonlinear optimization problem and can be solved using a reduced-gradient optimization algorithm, which is available in a number of numerical packages [21]. To increase the speed of convergence and the accuracy of the reduced-gradient algorithm, the gradient of the objective function (36) with respect to the parameters need to be computed. The gradients of (36) can be computed recursively using (30), as shown in [22].

The discussion of the previous section suggests formulation of the problem in an alternative, much simpler way. Instead of minimizing (36), it is possible to consider the objective function

$$
O_{\nu}=\sum_{h, k}\left(\nu_{h, k}\left(\lambda_{x}, \lambda_{y}\right)-\sum_{i, j} p_{i, j} d_{1_{i}}^{h} d_{2_{j}}^{k}\right)^{2}
$$

where $\nu_{h, k}\left(\lambda_{x}, \lambda_{y}\right)$ are computed from the moments $\mu_{h, k}$ of the image via (14). The simple expression of the gradient for (37) is shown in [22]. In the ideal optimal case where the image is generated by IFS parameters as in (6), if we find parameters 


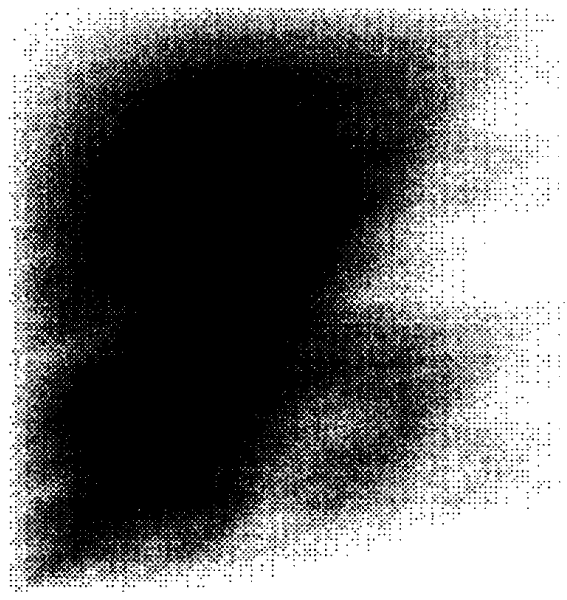

(a)

Fig. 11. Fractal Stroke: (a) Original; (b) $(6,7)$ approximation

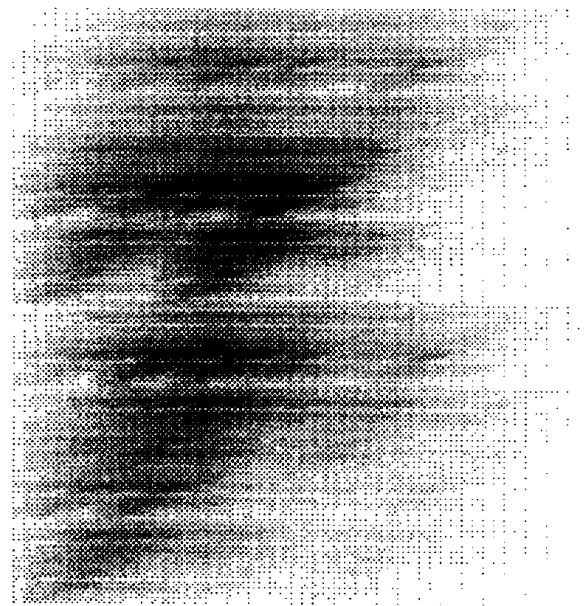

(a)

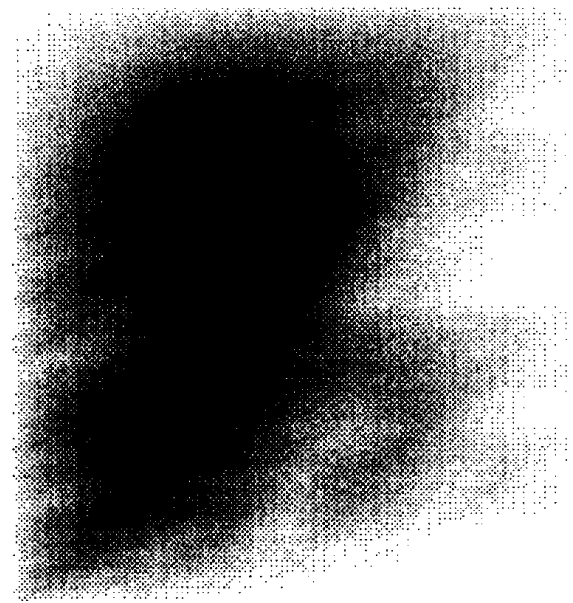

(b)

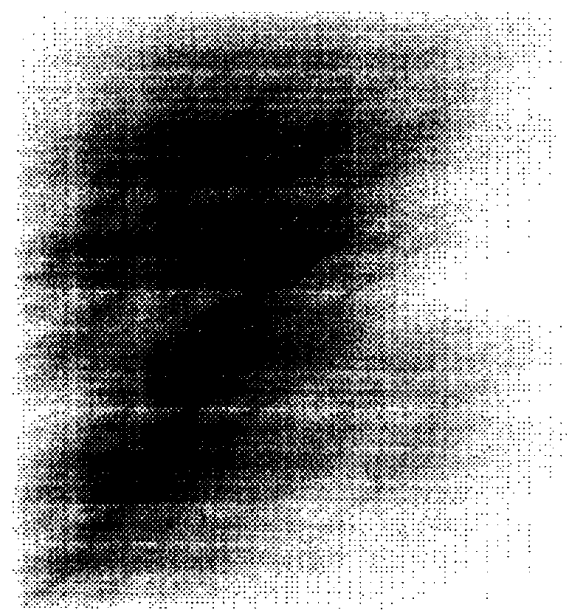

(b)

Fig. 12. Fractal Stroke: (a) $(4,4)$ approximation; (b) $(5,5)$ approximation

$\lambda_{x}, \lambda_{y} ; p_{i, j}, d_{1_{i}}, d_{2_{j}}$ for $i=1, \ldots, N_{1}, j=1, \ldots, N_{2}$ such that the objective function (37) is exactly zero, we would have $\tilde{\nu}_{h, k}=\nu_{h, k}$. To ensure the one-to-one correspondence between $\mu_{h, k}$ and $\nu_{h, k}, \mathcal{R}$ must be chosen such that there is an invertible relation between $\mu_{h, k}$ and $\nu_{h, k}$. As we will see in Section VI, a good choice is the triangular region of indexes $\{(h, k): h+k<L\}$ for some integer $L$. Note that for a fixed region of indices $(h, k)$ in which the nonlinear functions (36) and (37) are minimized, we can choose a different number of transformations for the IFS. Intuitively speaking, for a fixed region $\mathcal{R}$, the smaller the number of transformations, the more "overdetermined" the problem becomes. We will see effects of this in the approximation examples.

\section{EXAmPles of THE APPRoximation Procedure}

In this section, we present examples of the approximation procedure outlined in the previous section. We only consider a finite number of moments of the original image and compute a valid IFS of type (6) with the same moments. In Section VIA, we consider the approximation of IFS generated images, whereas in Section VI-B, we consider the approximation of real images. 


\section{A. Approximation of IFS-Generated Images}

Although our primary motivation in deriving the results in Section V has been to approximate arbitrary images, they can also be used in lower order approximations of IFSgenerated images with a large number of transformations. In this section, we apply the technique described in Section V to the approximation of IFS-generated images.

We begin with the application of the approximation procedure to the Snowflake of Fig. 3(a). As explained in Section IV, the Snowflake is obtained with five transformations, with three different displacement parameters both in the $x$ direction and the $y$ direction. Using the scheme of Section $\mathrm{V}$, we would need a $(3,3)$ order approximation to recover the parameters exactly where five transformations out of nine would have zero probability.

Fig. 8 shows a $(2,2)$ order approximation of the Snowflake. Due to the symmetry of the measure, its moments satisfy $\mu_{h, k}=\mu_{k, h}$. We arbitrarily chose the values $\lambda_{x}=\lambda_{y}=$ $0.739=\lambda_{\max }$ in the admissible range $U_{x}=U_{y}=[0,0.739]$ as the scaling parameters. The IFS parameters of the approximation are included in [22]. The IFS approximation matches eight moments of the original image, corresponding to the region $\mathcal{R}$ relative to a $(2,2)$ order approximation as defined in Section V. As seen, the appearance of the approximating image is quite different from the original one. A discussion of the properties of lower order moments can be found in [15]. In particular, it is noted there that the first- and second-order moments define an equivalent ellipse-like approximation of the original image.

Fig. 9(a) shows a $(3,3)$ order approximation of the Snowflake. The corresponding IFS is shown in Table I(b). Note that except for the scaling parameters, the displacement parameters and probabilities are closely related to the true values listed in Table I(a). Fig. 10 shows the smallest eigenvalue of the matrices $H_{2}^{(j)}, j=0,1,2$ as a function of $\lambda_{x}$. The corresponding matrices and eigenvalues for the $y$ direction are exactly equal to those for the $x$ direction due to the symmetry of the moment sequence noted above. As seen, there is only one interval $U_{x}=U_{y}=\left[0, \lambda_{\max }\right], \lambda_{\max }=0.333$ in which all of the three matrices are positive definite. We arbitrarily choose $\lambda_{x}=\lambda_{y}=0.25$ in $U_{x}=U_{y}=[0,0.333]$.

The $(3,3)$ order approximation recovers the Snowflake parameters exactly if we choose $\lambda_{x}=\lambda_{y}=\lambda_{\max }=0.333$ as expected. The reconstructed image is in Fig. 9(b). Thus, for a $(3,3)$ approximation, any value of $\lambda_{x}$ and $\lambda_{y}$ in the allowable range leads to an IFS measure that matches the moments of the original image in $\mathcal{R}$; the value $\lambda_{x}=\lambda_{y}=\lambda_{\max }=0.333$ results in the original snowflake, whereas $\lambda_{x}=\lambda_{y}=0.25$ results in a similar looking image.

Fig. 11(a) shows the Fractal Stroke that is computed with 25 transformations as listed in Table II(a). There are six different displacement parameters $d_{1_{i}}$ and seven different displacement parameters $d_{2_{j}}: \lambda_{x}=0.54$ and $\lambda_{y}=0.41$. In the approximation scheme of the previous section, this corresponds to 42 transformations, only 25 of which have nonzero probabilities. Fig. 12 shows a $(4,4)$ and a $(5,5)$ approximation of the Fractal Stroke with $\lambda_{x}=0.57, \lambda_{y}=0.29$. The values of

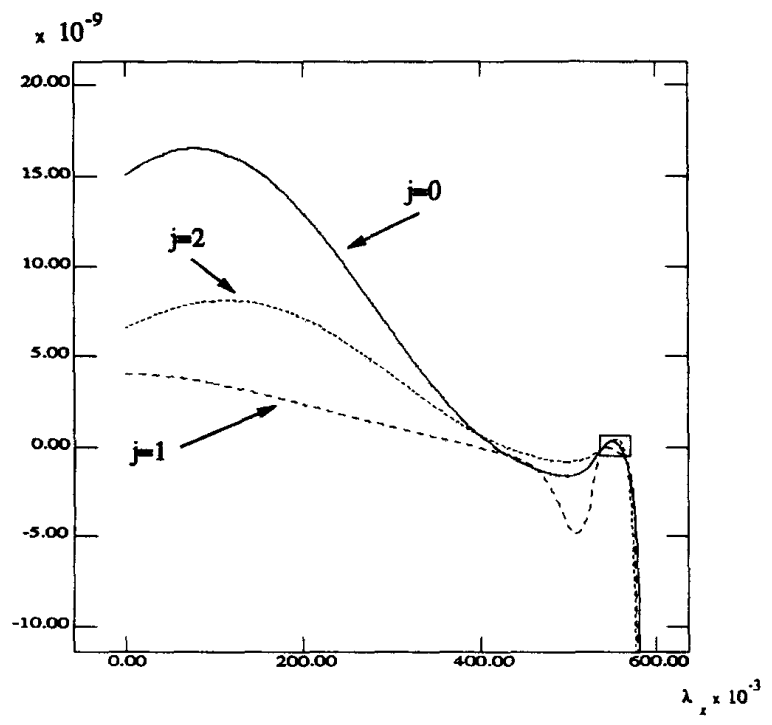

(a)

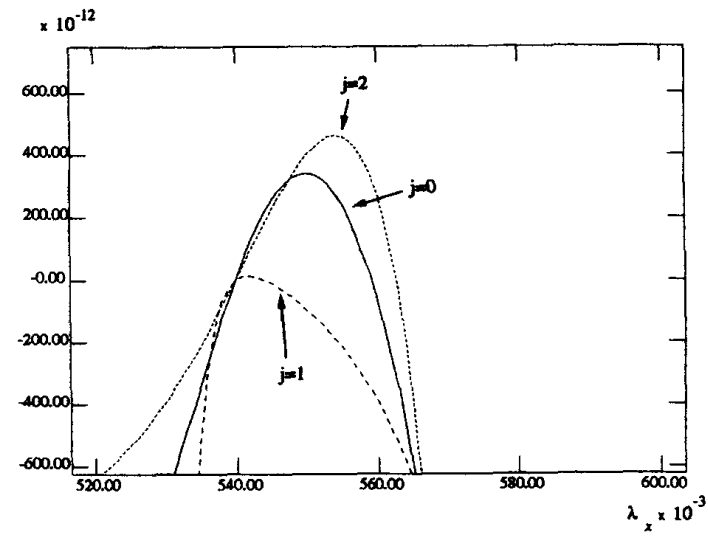

(b)

Fig. 13. (a) Smallest eigenvalue of $H_{5}^{(j)}, j=0,1,2$ versus $\lambda_{x}$ for a $(6,7)$ approximation; (b) expansion of the square in (a).

$\lambda_{x}$ and $\lambda_{y}$ were chosen in the allowable range following the arbitrary criterion of maximizing the sum $\lambda_{x}+\lambda_{y}$ in order to have an IFS that is less contractive. In each case, we find that there is only one interval of $\lambda_{x}$ and $\lambda_{y}$ satisfying conditions (33). The remaining IFS parameters of the approximations are included in [22]. Some of the probabilities for the $(5$, 5) approximation are zero to numerical precision, and the corresponding transformations can be eliminated from the IFS. As seen, the approximations become more similar to the original image as the number of transformations increases; in particular, the $(5,5)$ approximation seems to retain all the basic features of the original image.

In contrast to the $(4,4)$ and $(5,5)$ approximations, in the case of the $(6,7)$ approximation, the sets $U_{x}$ and $U_{y}$ are 
TABLE II

IFS Parameters of the Fractal Stroke: (a) Original; (b) $(6,7)$ Order Approximation with $O_{\mu}$ Optimization; (c) $(6,7)$ Order ApProximation with $O_{\nu}$ Optimization

\begin{tabular}{|c|c|c|}
\hline$d_{1 ;}$ & $d_{2_{i}}$ & $p_{i}$ \\
\hline 0.010 & 0.010 & 0.112 \\
\hline 0.010 & 0.100 & 0.020 \\
\hline 0.010 & 0.250 & 0.040 \\
\hline 0.010 & 0.400 & 0.060 \\
\hline 0.010 & 0.590 & 0.020 \\
\hline 0.180 & 0.010 & 0.030 \\
\hline 0.180 & 0.100 & 0.030 \\
\hline 0.180 & 0.250 & 0.050 \\
\hline 0.180 & 0.590 & 0.020 \\
\hline 0.310 & 0.400 & 0.050 \\
\hline 0.100 & 0.500 & 0.034 \\
\hline 0.180 & 0.400 & 0.025 \\
\hline 0.010 & 0.500 & 0.040 \\
\hline 0.200 & 0.590 & 0.040 \\
\hline 0.310 & 0.300 & 0.030 \\
\hline 0.310 & 0.500 & 0.030 \\
\hline 0.200 & 0.500 & 0.025 \\
\hline 0.200 & 0.100 & 0.025 \\
\hline 0.180 & 0.500 & 0.030 \\
\hline 0.310 & 0.590 & 0.038 \\
\hline 0.460 & 0.590 & 0.045 \\
\hline 0.460 & 0.100 & 0.045 \\
\hline 0.100 & 0.100 & 0.038 \\
\hline 0.100 & 0.590 & 0.025 \\
\hline 0.200 & 0.300 & 0.098 \\
\hline
\end{tabular}

(a)

\begin{tabular}{ccc}
\hline$d_{1_{i}}$ & $d_{2_{i}}$ & $p_{i}$ \\
\hline 0.000 & 0.000 & 0.146 \\
0.000 & 0.306 & 0.079 \\
0.000 & 0.117 & 0.074 \\
0.000 & 0.503 & 0.103 \\
0.110 & 0.306 & 0.105 \\
0.110 & 0.117 & 0.081 \\
0.110 & 0.503 & 0.149 \\
0.288 & 0.117 & 0.100 \\
0.288 & 0.503 & 0.159 \\
0.506 & 0.000 & 0.003 \\
\hline \multicolumn{2}{c}{$=0.695, \lambda_{y}=0.464$}
\end{tabular}

(b)

\begin{tabular}{ccc}
\hline$d_{\mathbf{1}_{\boldsymbol{i}}}$ & $\boldsymbol{d}_{\mathbf{2}_{i}}$ & $p_{\boldsymbol{i}}$ \\
\hline 0.011 & $\mathbf{0 . 0 0 0}$ & 0.100 \\
0.011 & $\mathbf{0 . 1 3 8}$ & 0.048 \\
0.011 & 0.326 & 0.037 \\
0.011 & 0.507 & 0.066 \\
0.147 & 0.000 & 0.050 \\
0.147 & 0.138 & 0.076 \\
0.147 & 0.326 & 0.091 \\
0.147 & 0.507 & 0.138 \\
0.292 & 0.138 & 0.099 \\
0.292 & 0.326 & 0.070 \\
0.292 & 0.507 & 0.114 \\
0.292 & 0.606 & 0.018 \\
0.482 & 0.000 & 0.022 \\
0.482 & 0.326 & 0.025 \\
0.482 & 0.507 & 0.025 \\
0.482 & 0.606 & 0.021 \\
\hline$\lambda_{x}=0.494, \lambda_{y}$ & $=0.449$
\end{tabular}

(c) actually the union of two disjoint intervals. Fig. 13(a) shows the smallest eigenvalue of $H_{5}^{(j)}, j=0,1,2$ for the sequence $\nu_{h, 0}$ as a function of $\lambda_{x}$. Fig. 13(b) shows a detail of Fig. 13(a) around the true value of the scaling parameter $\lambda_{x}=0.54$. As expected, the matrices become positive definite again after being nonpositive definite. A similar behavior holds for the eigenvalue corresponding to the matrices of the sequence $\nu_{0, k}$; the matrices become positive definite in an interval around $\lambda_{y}=0.41$.

By maximizing the value $\lambda_{x}+\lambda_{y}$ in the range of $\lambda_{x} \in$ $U_{x}, \lambda_{y} \in U_{y}$ that give positive probabilities, we obtain, to numerical precision, the correct values $\lambda_{x}=0.54$ and $\lambda_{y}=$ 0.41 and get the approximation of Fig. 11. The approximation recovers the original IFS exactly within numerical precision. Fig. 14 shows approximations obtained using the nonlinear optimization methods of Section V-C. In both cases, the initial conditions where $\lambda_{x}=\lambda_{y}=0.5$, and the displacement parameters in the $x$ and $y$ directions were initially set to be equally spaced in the interval $[0,1]$, i.e., $d_{1_{i}}=(i-$ 1) $/ N_{1}, i=1, \ldots, N_{1}$ (or equivalently $d_{1_{j}}=(j-1) / N_{2}, j=$ $1, \ldots, N_{2}$ ) for $N_{1}$ displacement parameters $d_{1_{i}}$ (or equivalently $N_{2}$ displacement parameters $d_{2_{j}}$ ). Probabilities were initially set to the same value $1 / N_{1} N_{2}$ for each transformation. In particular, Fig. 14(a) shows a $(6,7)$ approximation obtained by minimizing (36) on the region of indexes $\{(h, k), h+$ $k<10\}$. The IFS corresponding to a local minimum of the objective function has only ten transformations with nonzero probability and is shown in Table II(b). Fig. 14(b) shows a (6, 7) approximation obtained by minimizing (37) over the same region of indices as before. There are only 16 transformations with nonzero probability, as shown in Table II(c). The two images retain the basic features of the original one, but some striping effect in Fig. 14(a) and square-blocked artifacts in Fig. 14(b) are clearly visible, as a result of the obtained values for the displacement parameters and scaling factors in the two cases. The approximation of Fig. 14(b) required a much smaller computation time than that of Fig. 14(a) due to the simpler computation of the gradient vector in the nonlinear optimization procedure.

\section{B. Approximation of Real Images}

We have also applied our approximation procedure to real texture images. Fig. 15(a) shows a $256 \times 256$ digitized texture from the book [19]. Fig. 15(b) shows a $(8,3)$ approximation of the "Cheesecloth" of Figure 15a using the scheme of section V.A. For this case, we relax the conditions required to determine the intervals $U_{x}$ and $U_{y}$ by checking that only the matrices corresponding to $j=0$ in Lemma 1 are posi- 


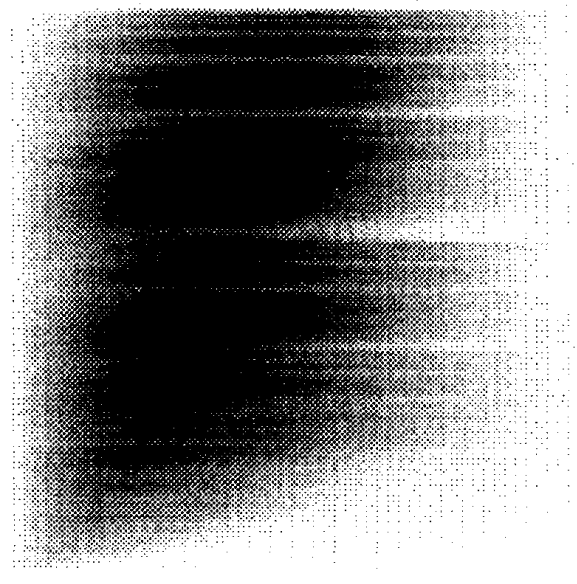

(a)

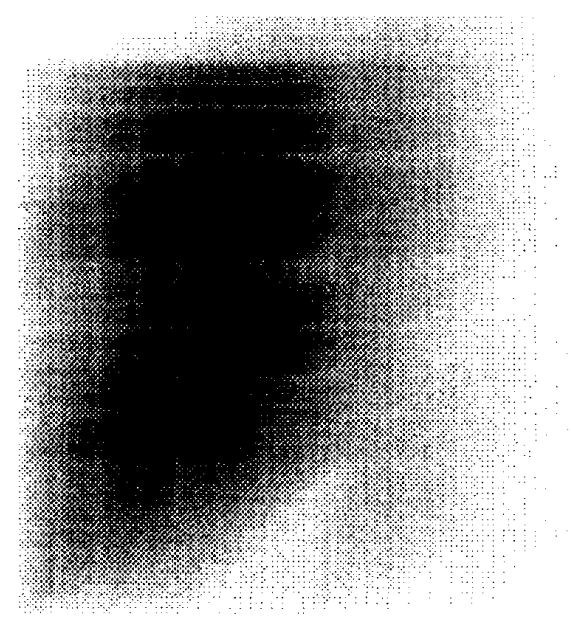

(b)

Fig. 14. Fractal Stroke: (a) $(6,7)$ approximation $\left(O_{\mu}\right.$ minimization); (b) $(6,7)$ approximation $\left(O_{\nu}\right.$ minimization).

tive definite. As noted, this guarantees that the displacement parameters are real numbers but not necessarily in the range $[0,1]$. The IFS for the $(8,3)$ approximation is in table 3a. As seen, the negative displacement parameters have very small magnitude, as expected because of the $[0,1) \times[0,1)$ support of the original image. We note that we represent the image with less than 37 parameters in total, corresponding to the set of probabilities, displacement parameters and $\lambda_{x}, \lambda_{y}$ of the IFS.

The approximating image matches well the general appearance of the original one. Note, in particular, the correspondence between dark regions in the original and approximated image. However, the correspondence between the exact position and the shape of the vertical features in the two images is only approximate. The values of $\lambda_{x}$ and $\lambda_{y}$ were chosen in the allowable ranges $U_{x}=[0,0.19]$ and $U_{y}=[0,0.508]$ to minimize the presence of artifacts. In particular, horizontal stripes are visible for certain values of $\lambda_{y}$. Starting from the pair of values that maximize $\lambda_{x}+\lambda_{y}$, some trial and error was necessary to determine the final values. Note that our chosen value $\lambda_{y}=0.33$ and the values of the three displacement parameters in the $y$ direction, i.e., $d_{2_{1}}=0.330, d_{2_{2}}=-0.004, d_{2_{3}}=0.664$, prevent horizontal stripes because the image replicas produced by the IFS transformations barely touch each other. Intuitively speaking, for $\lambda_{y}>0.33$, the replicas would have been overlapping each other, and for $\lambda_{y}<0.33$, there would have been gaps between the replicas, thus resulting in horizontal stripes in both cases. Increasing the number of transformations did not improve the approximation. Blank regions and white stripes began to appear in the approximation. A discussion of this effect is delayed to the conclusion in Section VII.

Fig. 15(c) is obtained with an IFS whose parameters are computed by optimizing (36) over the region $\mathcal{R}$ corresponding to indexes $\{(h, k): h+k<10\}$. Initial conditions were $\lambda_{x}=\lambda_{y}=0.5$ with equally spaced displacement parameters in $[0,1]$ and equal probability for each transformation. The image corresponds to an IFS with eight $d_{1_{i}}$ 's and three $d_{2_{j}}$ 's. Only 12 out of the 24 possible transformations have nonzero probabilities, and only six $d_{1_{i}}$ 's appear in the IFS shown in Table III(b). In this case, there is a correspondence between dark regions in the original and approximating image. The approximating image appears smoother than the one in Fig. 15(b) obtained with the exact matching procedure.

Fig. 16(a) shows the $256 \times 256$ digitized image "Water" from [19]. Fig. 16(b) shows a $(3,3)$ approximation of the image obtained with the procedure of Section V-A. The final values of $\lambda_{x}$ and $\lambda_{y}$ were obtained after some trial and error to minimize vertical and horizontal stripes. Only six of the transformations result to have nonzero probability. Fig. 16(c) shows a $(3,8)$ approximation based on the minimization of (36) on the region of indexes $\{(h, k): h+k<10\}$. Only eight transformations have nonzero probability, and only three of the eight displacement parameters $d_{2}$ play a role in the IFS. Initial conditions were set to the values obtained for the $(3,3)$ approximation of Fig. 16(b); additional transformations for the $(3,8)$ approximation were initially assigned zero probabilities.

Horizontal stripes are clearly seen in the approximations of Fig. 16(b) and (c) that are not present in the original image in Fig. 16(a). The image "Water" is almost a uniform luminance gradient from black to white in the vertical direction and is not inherently "self-similar." The approximating images retain the main feature of the original image, i.e., the vertical variation from a dark to a light region. However, horizontal stripes are present due to the characteristics of the IFS formalism, in which the resulting image is made up with overlapping scaled copies of the whole image. In particular, replicas of the dark region at the bottom of the image must be visible in the $(x, y)$ position corresponding to the displacement parameters. 


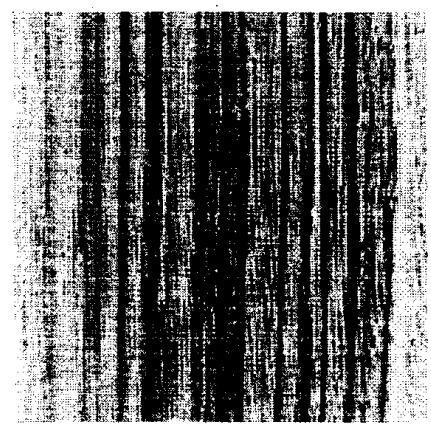

(a)

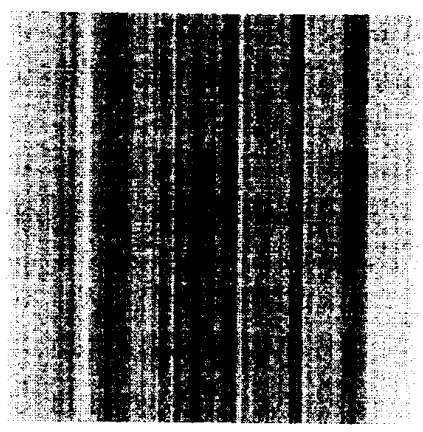

(b)

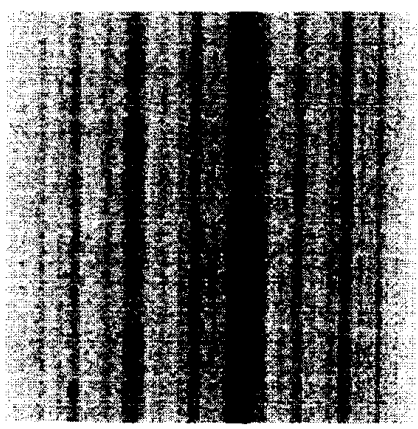

(c)

Fig. 15. "Cheesecloth": (a) Original; (b) $(8,3)$ approximation; (c) $(6,3)$ approximation with $O_{\mu}$ minimization.

TABLE III

IFS for the $(8,3)$ Approximation of the CheEsecloth with (a) Exact Moment Matching, (b) Overdetermined Moment Matching via $O_{\mu}$ Optimization and, (c) $(5,2)$ Order Approximation of "Raffia"

\begin{tabular}{ccc}
\hline$d_{1_{i}}$ & $d_{2_{i}}$ & $p_{i}$ \\
\hline 0.532 & 0.330 & 0.058 \\
0.532 & -0.004 & 0.058 \\
0.532 & 0.664 & 0.061 \\
0.199 & 0.330 & 0.055 \\
0.199 & -0.004 & 0.052 \\
0.199 & 0.664 & 0.055 \\
0.108 & 0.330 & 0.022 \\
0.108 & -0.004 & 0.021 \\
0.108 & 0.664 & 0.021 \\
-0.003 & 0.330 & 0.044 \\
-0.003 & -0.004 & 0.039 \\
-0.003 & 0.664 & 0.040 \\
0.367 & 0.330 & 0.071 \\
0.367 & -0.004 & 0.068 \\
0.367 & 0.664 & 0.066 \\
0.687 & 0.330 & 0.057 \\
0.687 & -0.004 & 0.051 \\
0.687 & 0.664 & 0.051 \\
0.815 & 0.330 & 0.038 \\
0.815 & -0.004 & 0.031 \\
0.815 & 0.664 & 0.040 \\
\hline$\lambda_{x}=0.19, \lambda_{y}=0.33$
\end{tabular}

(a)

For example, in Fig. 16(b), a horizontal line is present in correspondence to the displacement parameter $d_{2_{1}}=0.495$. The effect is particularly evident because of the form (6) of the IFS we consider, which tends to result in approximating images with a square-blocked appearance. This is indicative of the fact that our proposed approximation scheme is appropriate only for a specific class of images that inherently possess a certain degree of "self-similarity" and that the result of the approximation can be poor for other images.

\begin{tabular}{ccc}
\hline$d_{1_{i}}$ & $d_{2_{i}}$ & $p_{i}$ \\
\hline 0.006 & 0.000 & 0.133 \\
0.006 & 0.364 & 0.009 \\
0.006 & 0.499 & 0.145 \\
0.000 & 0.000 & 0.105 \\
0.000 & 0.499 & 0.090 \\
0.357 & 0.000 & 0.049 \\
0.357 & 0.499 & 0.038 \\
0.460 & 0.000 & 0.204 \\
0.460 & $\mathbf{0 . 4 9 9}$ & 0.037 \\
0.461 & 0.499 & 0.041 \\
0.467 & 0.364 & 0.011 \\
0.467 & $\mathbf{0 . 4 9 9}$ & $\mathbf{0 . 1 3 7}$ \\
\hline$\lambda_{x}=0.538, \lambda_{y}=0.5$
\end{tabular}

\begin{tabular}{ccc}
\hline$d_{1_{i}}$ & $d_{2_{i}}$ & $p_{i}$ \\
\hline 0.324 & 0.499 & 0.119 \\
0.324 & 0.000 & 0.143 \\
0.155 & 0.499 & 0.068 \\
0.155 & 0.000 & 0.062 \\
0.000 & 0.499 & 0.085 \\
0.000 & 0.000 & 0.116 \\
0.554 & 0.499 & 0.131 \\
0.554 & 0.000 & 0.100 \\
0.776 & 0.499 & 0.085 \\
0.776 & 0.000 & 0.091 \\
\hline \multicolumn{2}{l}{$\lambda_{x}=0.227, \lambda_{y}=0.5$}
\end{tabular}

(c)

(b)

Fig. 17(a) shows the image "Raffia" from [19]. Fig. 17(b) shows a $(5,2)$ approximation of the image. The approximation is obtained by combining the exact matching and optimization procedures. In particular, the approximation is obtained by minimizing (36) on the region of indexes $\{(h, k): h+k<12\}$, starting from initial conditions given by the exact matching procedure for the same approximation order. The values of $\lambda_{x}$ and $\lambda_{y}$ in the exact matching procedure were chosen in the admissible region in such a way as to minimize the presence 


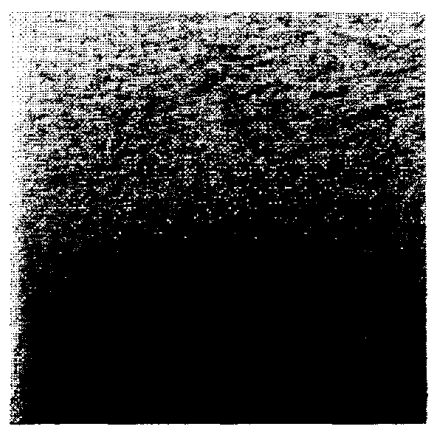

(a)

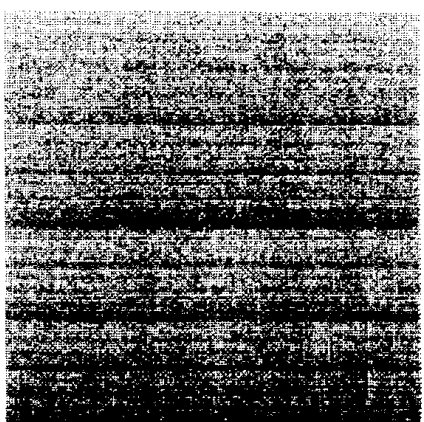

(b)

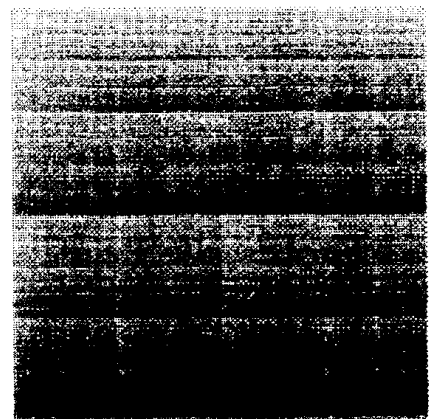

(c)

Fig. 16. "Water": (a) Original; (b) $(3,3)$ approximation; (c) $(3,8)$ approximation with $O_{\mu}$ minimization.

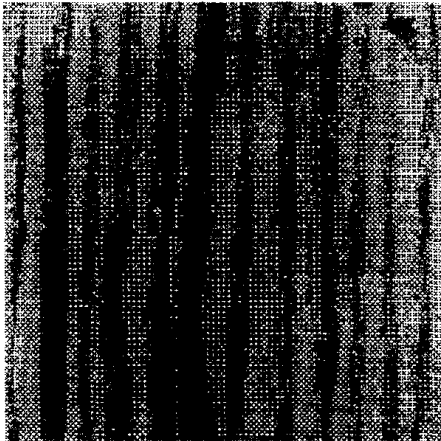

(a)

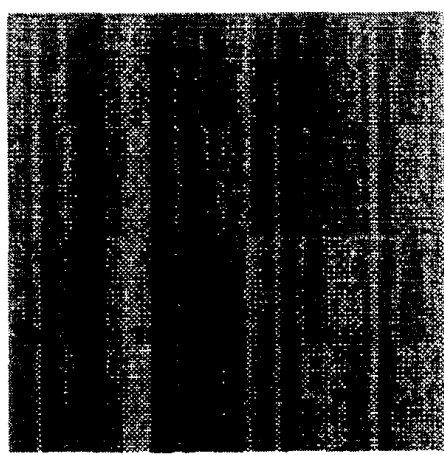

(b)

Fig. 17. (a) "Raffia"; (b) $(5,2)$ approximation of "Raffia."

of stripes. The IFS of the approximation is in Table III(c). As seen, the approximation retains the features of the original image only approximately; some square-blocked artifacts are clearly noticeable.

\section{CONCLUSION}

We considered the problem of determining the IFS parameters for a class of 2-D fractal signals by applying the wavelet transform and the moment method. The method is applicable to images that are generated by 2-D homogeneous IFS and exploits the self-similarity of fractal objects along different scales.

The extension of our method to the case of a general IFS is not immediate. This is due to the fact that there is no simple recursive expression for moments in the general case [16]. In addition, the application of the wavelet transform method to the case of different matrices $Q_{i}$ in (2), even if the diagonal structure is maintained, would not permit us to determine all of the different scaling parameters but only those corresponding to transformations $w_{i}$ whose fixed points are easily determined; see the discussion in Section III-A-1.

An approximation procedure has also been suggested for the case of arbitrary images not necessarily generated by IFS parameters; the original image is known via its moments, and the reconstruction process aims at matching those moments. In principle, the power of the moment approach is significant because of the one-to-one correspondence between 2-D compact support measures and the sequence of their moments [12]. This implies that measures with the same infinite sequence of 2-D moments coincide. From a practical standpoint, the coincidence of a finite set of moments does not guarantee in general that the two images look alike; see, for instance, [15]. In particular, the appearance of the approximating image can be different from that of the original image, even if the number of matched moments is significant, since the moments only give average information on the original image. This implies that the resulting IFS image can look different from the original one. 
Indeed, following the method of Section $\mathrm{V}$, we could find a 2-D atomic measure $\tilde{\nu}$ whose moments match the moments $\mu_{h, k}$ of the original image $\mu$ directly instead of $\nu_{h, k}$. The "geometrical" appearance of the original nonatomic measure $\mu$ and of the approximating atomic measure would be very different. As a matter of fact, when we increase the number of transformations of the IFS, we empirically find that the allowable range of values of $\lambda_{x}$ and $\lambda_{y}$ becomes smaller, and typically closer to zero, as suggested by Theorem 1 . As a consequence, the resulting highly contractive IFS will have an invariant measure that becomes increasingly similar to an atomic one, and its visual appearance can be very different from the original image. In addition, numerical instability arises as we increase the number of transformations for the IFS, as noted in [11] and [10] for the 1-D case. The potential usefulness of the approximation approach of Section V derives from the fact that we actually approximate images with fractal measures. As evidenced by Figs. 15-17, the greater richness of the fractal representation should provide a powerful tool for the approximation of fractal-like images.

Future work involves the extension of our results to more general classes of IFS and the applicability of IFS techniques to the approximation of various regions within a real image.

\section{REFERENCES}

[1] M. Barnsley, Fractals Everywhere. Boston, Academic, 1988.

[2] J. H. Elton, "An ergodic theorem for iterated maps," Ergodic Theory Dynamical Syst., vol. 7, pp. 481-488, 1987.

[3] I. Daubechies, "The wavelet transform, time-frequency localization and signal analysis," IEEE Trans. Inform. Theory, pp. 961-1005, Sept. 1990

[4] J. M. Combes, A. Grossman, and P. Tchamitchian, Wavelets. Berlin: Springer, 1989

[5] G. C. Freeland and T. S. Durrani, "IFS fractals and the wavelet transform," in Proc. 1990 ICASSP, pp. 2345-2348.

[6] F. Argoul, A. Arneodo, J. Elezgaray, G. Grasseau, and R. Murenzi, "Wavelet analysis of the self-similarity of diffusion-limited aggregates and electrodeposition clusters," Phys. Rev. A, vol. 41, no. 10, May 15, 1990.

[7] A. Arneodo, G. Grasseau, and M. Holschneider, "Wavelet transform of invariant measures of some dynamical systems," in Wavelets (J. M. Combes, A. Grossman, P. Tchamitchian, Eds.). Berlin: Springer, 1989.

[8] M. F. Barnsley and S. Demko, "Iterated function systems and the global construction of fractals," Proc. R. Soc. Lond., A 399, pp. 243-275, 1985.

[9] C. R. Handy and G. Mantica, "Inverse problems in fractal construction: Moment method solution," Physica D, vol. 43, pp. 17-36, 1990.

[10] S. Abenda and G. Turchetti, "Inverse problem for fractal sets on the real line via the moment method," Il Nuovo Cimento, vol. 104 B, no. 2, Aug. 1989.

[11] S. Abenda, "Inverse problem for one-dimensional fractal measures via iterated function systems and the the moment method," Inverse Problems, vol. 6, 1990.

[12] J. A. Shohat and J. D. Tamarkin, The Problem of Moments. New York: Amer. Math. Soc., 1943
[13] J. H. Elton and Z. Yan, "Approximation of measures by Markov processes and homogeneous affine iterated function systems," Constructive Approximation, vol. 5, pp. 69-87, 1989.

[14] D. Bessis and S. Demko, "Stable recovery of fractal measures by polynomial sampling," Physica $D, 47$, pp. 427-238, 1991.

[15] M. R. Teague, "Image analysis via the general theory of moments," $J$. Opt. Soc. Amer., vol. 70, no. 8, pp. 920-930, Aug. 1980.

[16] E. R. Vrscay and C. J. Roehrig, "Iterated function systems and the inverse problem of fractal construction using moments," in Comput Math. (E. Kaltofen and S. M. Watt, Eds.). New York, Springer-Verlag, 1989.

[17] G. Strang, Linear Algebra and Its Applications. New York, Academic

[18] A. G. Baker, Jr. and P. G.-Morris, "Padé Approximants," in Encyclopedia of Mathematics and Its Applications, 1981, vol. 13-14.

[19] P. Brodatz, Textures-A Photographic Album for Artists and Designers. Dover: New York, 1966

[20] Wood and Wood Grains-A Photographic Album for Artists and Designers. Dover: New York, 1971.

[21] B. A. Murtagh and M. A. Saunders, "MINOS 5.1 User's Guide," Tech. Rep. SOL 83-20R, Stanford Univ., 1987.

[22] R. Rinaldo, Master's Thesis, Univ. of California, Berkeley, Dec. 1992.

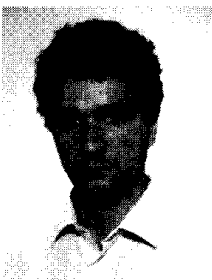

Roberto Rinaldo obtained the Laurea degree in electrical engineering in 1987 from the University of Padova, Padova, Italy, with honors and the medal for the highest graduation score. He received the M.S. degree from the University of California at Berkeley and the Doctorate degree from the University of Padova in 1992. Since 1993 , he has been with the Dipartimento di Elettronica o Informatica of the University of Padova, where he is currently a Ricercatore in Communications and Signal Processing. His interests are in the field of multidimensional signal processing, video signal coding, fractal theory, and image coding.

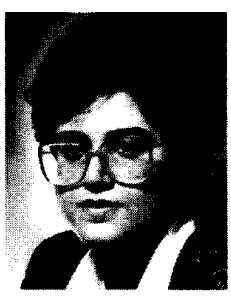

Avideh Zakhor received the B.S. degree from California Institute of Technology, Pasadena, and the S.M. and Ph.D. degrees from Massachusetts Institute of Technology, Cambridge, all in electrical engineering, in 1983, 1985, and 1987 respectively.

In 1988, she joined the Faculty at the University of California at Berkeley, where she is currently Assistant Professor in the Department of Electrical Engineering and Computer Sciences. Her research interests are in the general area of signal processing and its applications to images and video and biomedical data. She has been a consultant to a number of industrial organizations and holds four U.S. patents.

Dr. Zakhor was a General Motors scholar from 1982 to 1983, received the Henry Ford Engineering Award and Caltech Prize in 1983, was a Hertz fellow from 1984 to 1988, received the Presidential Young Investigators (PYI) award, IBM junior faculty development award, and Analog Devices junior faculty development award in 1990, and the Office of Naval Research (ONR) young investigator award in 1992. She is currently an Associate Editor for IEEE TRANSACTIONS ON IMAGE PROCESSING and a member of the technical committee for multidimensional digital signal processing. 